\title{
The peculiar B[e] star HD $45677^{\star}$
}

\section{Photometric behaviour and spectroscopic properties}

\author{
D. de Winter ${ }^{1,2}$ and M.E. van den Ancker ${ }^{1}$ \\ 1 Astronomical Institute "Anton Pannekoek", University of Amsterdam, Kruislaan 403, 1098 SJ Amsterdam, The Netherlands \\ 2 Dpto. Física Teórica, C-XI, Facultad de Ciencias, Universidad Autónoma de Madrid, Cantoblanco, E-28049 Madrid, Spain
}

Received October 31, 1995; accepted June 3, 1996

\begin{abstract}
The photometric behaviour of the peculiar $\mathrm{B}[\mathrm{e}]$ star HD 45677 over the last 25 years is investigated. We conclude that the photometric variations $\left(V=7^{\mathrm{m}} 22-8 \mathrm{~m} 85\right)$ on such a time scale can be well explained by obscurations, possibly due to large $(>1 \mu \mathrm{m})$ circumstellar (CS) dust grains which were created after an explosive event around 1950. Intermediate time scale variations are also identified and can be well explained by infall of CS material.

Evidence is also found for smaller, pulse-like, amplitude variations. The time scale of this "flickering" ranges from days down to hours and is explained by instabilities in accretion flows towards HD 45677. The accretion mechanisms can be the origin of the hypothesized existence of
\end{abstract} a bipolar flow.

HD 45677, seen edge-on, shows evidence for the presence of a circumstellar disk. The significant accretion flows in this disk probably increased some time after the 1950 event, either due to a fall-back of part of the ejected material by a blow-out around 1950 or either due to the explosive dissociation of a large cometary-like body. Dynamic effects of such infalling and outflowing material close to the stellar surface are also detected by high resolution spectroscopy in the $\mathrm{H} \alpha$ and He I profiles. These short time scale variations are also seen in the cool material as detected by the variations in the violet part of the $\mathrm{Na} I$ profiles, which are probably due to collisions of the outflowing material with the outer disk.

The presence of a disk is often indicated and here by the emission of the sodium lines with a strong absorption component at the systemic velocity (about $20 \mathrm{~km} \mathrm{~s}^{-1}$ ). Most detected lines, except $\mathrm{H} \alpha$, are well centered at this velocity. Apart from the red [SII] lines, all other nebular lines as well as many other emission lines, including the Fe II emission spectrum, were seen in spectra taken far

Send offprint requests to: D. de Winter (dolf@astro1.ft.uam.es) * Based on observations collected at the European Southern Observatory, La Silla, Chile, and on data collected in the longterm photometry of variables programme at ESO. before and after the 1950 event as well as in the latest spectra. So, the situation of the gaseous stellar environment seems to be stable in spite of the large photometric variations. The FeII emission lines are double peaked with a velocity separation of about $32 \mathrm{~km} \mathrm{~s}^{-1}$. This could mean that the inner disk material, in which these lines are thought to be formed, rotates with about $16 \mathrm{~km} \mathrm{~s}^{-1}$. In our 1992 data this rotation velocity seems to range up to $30 \mathrm{~km} \mathrm{~s}^{-1}$, which could be due to material accelerated by the 1950 event.

We discuss in this paper the evolutionary status of HD 45677. Because of its rather isolated position in the sky and because of the very fast evolution of a B2 type star, we think that HD 45677 could be young, but not in the sense of being a pre-main sequence object. Options like an evolved object such as a LBV or PN are not suitable because of the probable luminosity class III, IV or $\mathrm{V}$ and its rather cool central source, respectively. HD 45677 shows no evidence of any companion. The slight possibility of it being symbiotic can be added as well as instabilities in its unknown post-main sequence phase and the option that HD 45677 could be a hot post-AGB star.

Key words: circumstellar matter — stars: emission-line — stars: HD 45677 — stars: variables — infrared: stars

\section{Introduction}

The star HD 45677 ( $=$ FS CMa, $\alpha_{2000}=06^{\mathrm{h}} 28^{\mathrm{m}} 17^{\mathrm{s}} \cdot 4$, $\delta_{2000}=-13^{\circ} 03^{\prime} 10^{\prime \prime}$ ), is a well studied peculiar Be star with a strong infrared excess. Several attempts were made to classify peculiar Be stars in homogeneous groups. Wackerling (1970) indicated a variety of hot stars with abnormal spectra as BQ, and additionally those with forbidden lines were classified as BQ[ ] (Ciatti et al. 1974). Stars with characteristics such as HD 45677, were named B[e] stars 
(Allen \& Swings 1976) referring to the presence of forbidden lines, e.g. [OI] and [FeII], besides permitted emission lines of which the Balmer lines can be very strong with sometimes indications of stellar winds. Because of their absence in star-forming regions it is often believed that these objects are evolved objects, e.g. being peculiar Be-stars, being in certain stages of the early evolution of planetary nebulae (PN) or being symbiotic (Zickgraf \& SchulteLadbeck 1989; Halbedel 1989). Evidence for the evolved state of such objects are the $\mathrm{B}[\mathrm{e}]$ supergiants as located in the Magellanic Clouds (Zickgraf \& Schulte-Ladbeck 1989). Most known galactic B[e] stars have unknown luminosity class but the ones with reasonably well-determined luminosity classes seem to be quite heterogeneous in it. Their exact evolutionary state is therefore doubtful. Especially interesting is the case of HD 45677, in the past literature often called the "prototype" of the B[e] stellar group but also suggested to be a young stellar object.

The spectral classification of HD 45677 is difficult because of the presence of strong emission lines in its spectrum. It is generally agreed that its spectral type is B2 (e.g. Allen 1973; Feinstein et al. 1976). Its luminosity class however is still uncertain, most probably it is III, IV or V. In the recent catalogue of members and candidate members of the Herbig Ae/Be stellar group (Thé et al. 1994), HD 45677 is classified as an extreme emission line object (EELO). This term only indicates the spectral properties.

Recently, Sitko et al. (1994) argued that the difference in two photometric datasets of HD 45677, obtained in 1980 and in 1992, could be explained by changes in the level of stellar obscuration by circumstellar dust. This dust then acts as a grey absorber, being effective from the UV to the near infrared. Support for this explanation is that they observed a small decrease in total infrared flux, which is then caused by a decrease of the total mass of the star's circumstellar dust envelope. Interesting is that they obtained datasets consisting of UV to IR data at stages when HD 45677 was at minimum brightness (1980) and in a much brighter one (1992). However, their observational interval to obtain these multiwavelength datasets was about 1 month. It is known that HD 45677 varies significantly in brightness on such a time scale. It can therefore be questioned whether the deviations of their differential data from a grey absorber model are real or are due to photometric variations between their data at different wavelengths. Furthermore, modelling of the UV extinction curve of HD 45677 with a multi-scattering nonspherical circumstellar dust shell by Voshchinnikov et al. (1995) showed that it is possible to obtain a flat extinction curve using smaller dust particles, but with a nonstandard chemical composition, as well.

Schulte-Ladbeck et al. (1993) demonstrated that the position angle of the intrinsic polarization in the UV is flipped $90^{\circ}$ compared to that in the visual. This observation can be perfectly described by two polarization components, one from a bipolar flow with small optical depth, and one from the equatorial disk of large optical depth. At short wavelengths the direct starlight is blocked by the equatorial disk and the polarization of the scattered light from the polar lobs will dominate. Polarization from the equatorial disk will dominate at long wavelengths. The polarization flip occurs where both contributions are equal. Furthermore, the observed UV intrinsic polarization displays a stronger increase than in the red. This observation is explained by two distinct grain-size distributions in both regions or by the variation of the optical depth in the disk with wavelength which effects polarization values for both regions as well. Further support for the first possibility comes from the IRAS LRS spectrum of HD 45677, in which the $9.8 \mu \mathrm{m}$ silicate feature, seen superimposed on a strong dust continuum, is broadened between 9 and $11 \mu \mathrm{m}$ (Fajardo-Acosta \& Knacke 1995). The models by Simpson (1991) demonstrate that such broadening of the silicate emission can indeed be due to the presence of different sizes of dust grains.

The presence of high-velocity $\left(+200\right.$ to $\left.+400 \mathrm{~km} \mathrm{~s}^{-1}\right)$, accreting gas in all IUE spectra between 1979 and 1992 led Grady et al. (1993) to extend this picture by suggesting that HD 45677 may be a massive Herbig Be star with an actively accreting gaseous circumstellar disk, viewed nearly edge-on. The polarization data would then be explained by some of the UV light being scattered in a bipolar nebula oriented orthogonally to the dust disk. However, recent observations by Oudmaijer \& Drew (1995) showed that the intrinsic polarization in $\mathrm{H} \alpha$ is also flipped, but only by about $30^{\circ}$ compared to that in the continuum. This would indicate the presence of a significant amount of gas located intermediate between the bipolar flow and the disk. Grady et al. (1994) noted that the prominence of the pronounced near-UV emission, mainly due to Fe II and Mg II, has decreased with increasing system light.

Although there is no obvious nebulosity visible around HD 45677 (Swings 1973) and the star is not located in or near a star forming region, it has been suggested that HD 45677 is a young stellar object (age $\leq 10^{8}$ year) surrounded by a dust shell left over from star formation (Sorrell 1989). This view is supported by the polarization measurements of Coyne \& Vrba (1976), who concluded that HD 45677 is surrounded by a ring of patchy clouds of dust with an inner radius of $45 \mathrm{AU}$, which also explains the strong infrared-excess. Another suggestion for the infrared excess of HD 45677 is a cool companion. This hypothetical companion has been suggested to be a cool giant (Ciatti et al. 1974), or an infrared object similar to the BecklinNeugebauer object (Low et al. 1970). Searches for CO (1-0) (Nyman et al. 1992), $18 \mathrm{~cm} \mathrm{OH}$ maser (Le Squeren et al. 1992), and $\mathrm{SiO}$ maser (Dickinson et al. 1978) emission from HD 45677 have been made, but all detections were negative. Since the recent $\mathrm{CO}$ and $\mathrm{OH}$ surveys were quite deep, and HD 45677 is much brighter than most objects that were detected, it is likely that the shell of HD 45677 is either too thin or too small to produce such 
emission or that the mass loss has been started recently. Therefore, it seems unlikely that HD 45677 is an evolved post-AGB OH/IR-object.

In this paper we will discuss the photometric and spectroscopic behaviour of HD 45677 over the last 25 years, based on both newly obtained data and data from the literature. Photometric variability from the UV to near infrared wavelength ranges on long $(\approx 40$ years), intermediate (a few months), and short (hours) time scales will be discussed and explained. Furthermore, we will discuss the star's spectral characteristics, and discuss and explain observed short-term spectroscopic variations in the $\mathrm{Na}$ I $D$ and $\mathrm{H} \alpha$ lines. However, a more detailed description of the implications of these data will be given in a forthcoming paper (Israelian et al. 1996). Finally, some remarks will be added to the discussion on the evolutionary phase of HD 45677.

\section{The observations}

\subsection{Photometry}

New and unpublished photometric data of HD 45677 in the Walraven $W U L B V$, Strömgren uvby, Johnson/Cousins $U B V(R I)_{\mathrm{C}}$ and ESO JHKLM system are listed in an accompanying paper (de Winter et al. 1996; Paper I) and will be analyzed and discussed in this paper.

\subsection{Spectroscopy}

Several new low resolution CCD spectra of HD 45677 were taken at La Silla using the Boller and Chivens spectrograph mounted on the ESO $1.52 \mathrm{~m}$ telescope. Details of the observations are given in Table 1 . In order to get a sufficiently good $\mathrm{S} / \mathrm{N}$ for weaker emission lines it was necessary to have the strength of $\mathrm{H} \alpha$ exceed the CCD saturation limit. To be able to measure the relative strength of $\mathrm{H} \alpha$ second, shorter, exposures during the same two December nights were taken in which $\mathrm{H} \alpha$ was not saturated. A combined spectrum of the $1992 \mathrm{~d}, \mathrm{e}, \mathrm{f}$ and $1992 \mathrm{~g}$ spectra is presented in Fig. 4.

To reveal the $\mathrm{H} \alpha$ profile and to test for short time scale variations two intermediate resolution spectra, Fig. 5, centered around $\mathrm{H} \alpha$, were obtained. These observations, see Table 1, were made with the Boller and Chivens spectrograph mounted on the ESO $1.52 \mathrm{~m}$ telescope at La Silla. The detector for all the low and intermediate resolutions taken spectra was a Ford Aerospace $2048 \times 2048$ pixel CCD, with a $15 \times 15 \mu \mathrm{m}$ pixel size.

To detect short time scale variations, HD 45677 was followed day to day between the $8^{\text {th }}$ and the $14^{\text {th }}$ of October 1993 by using the CAT/CES combination at La Silla. This combination was used to obtain high resolution spectra in the $\mathrm{H} \alpha$ and $\mathrm{NaI} D$ wavelength ranges, which are presented in Figs. 9 and 10. Further high resolution spectra, in the $\mathrm{H} \alpha$, Ca II $K$, and [O I] $6300 \AA$ wavelength ranges
(Figs. 6-8), were obtained in January 1994. During all observations, the detector was a $1024 \times 640$ RCA SID 006 high resolution CCD, with a pixel size of $15 \times 15 \mu \mathrm{m}$. All the CAT/CES observations were controlled remotely from ESO Headquarters, Garching bei München, Germany.

All spectra were reduced using the MIDAS software running under SUN/OS at ESO headquarters. For optimal extraction of spectral data from the CCD images the optimal extraction algorithm by Horne (1986) was applied. After reduction, the low-resolution spectra were combined into one single, completely unsaturated, spectrum, with spectrophotometric quality. The new spectral data are shown in Figs. 4-10. Additional details of all spectroscopic observations are listed in Table 1.

\subsection{Imaging}

Images of HD 45677 were obtained, under reasonable $\left(1{ }^{\prime \prime} 1-1{ }^{\prime \prime} 6\right)$ seeing conditions, with the Dutch $92 \mathrm{~cm}$ telescope at La Silla. Two observing runs were used, on the $10^{\text {th }}$ and $14^{\text {th }}$ of February 1993 , and on the $7^{\text {th }}$ of August 1993. The images were reduced using MIDAS, running on a VAX cluster at the University of Amsterdam. On all of the images, taken through $B, V, R$, Gunn $i$, Gunn $z$, wideband $\mathrm{H} \alpha$, narrowband $\mathrm{H} \alpha$ and narrowband [OI] $\lambda 6300$ filters, HD 45677 was indistinguishable from a stellar point source. No nebulosity of emission or reflection nature were visible. By checking POSS (Palomar Observatory Sky Survey) plates, searching for significant changes of the number of stellar objects in the surroundings of HD 45677, no dark region could be marked.

\section{Analysis of the photometric behaviour}

Together with the newly taken and previously unpublished data in Paper I, we collected all available visual photometric data from the literature. An overview of all visual photometry of HD 45677 used in our analysis is given in Table 2. As described in Paper I, these data were transformed, as good as possible, to values in similar bands of the "standard" Johnson $U B V$ and Strömgren uvby photometric systems.

The photographic magnitudes from Swings \& Swings (1972) are comparable to the visual magnitude at the time the first photoelectric measurements were taken, $B-V \approx 0$ m 0 (Mendoza 1958; Wampler 1968 in Swings \& Allen 1971). A different effective wavelength of the photographic and photoelectric measurements will therefore not cause a large systematic difference between $m_{\mathrm{pg}}$ and $V$. For the transformation of $U B V$ data of the Geneva system to those of the Johnson system we derived the following formulae from the absolute calibrations of both systems given by Schmidt-Kaler (1982) and by Rufener \& Nicolet (1988):

$U_{\mathrm{J}}=U_{\mathrm{G}}-0.305$ 

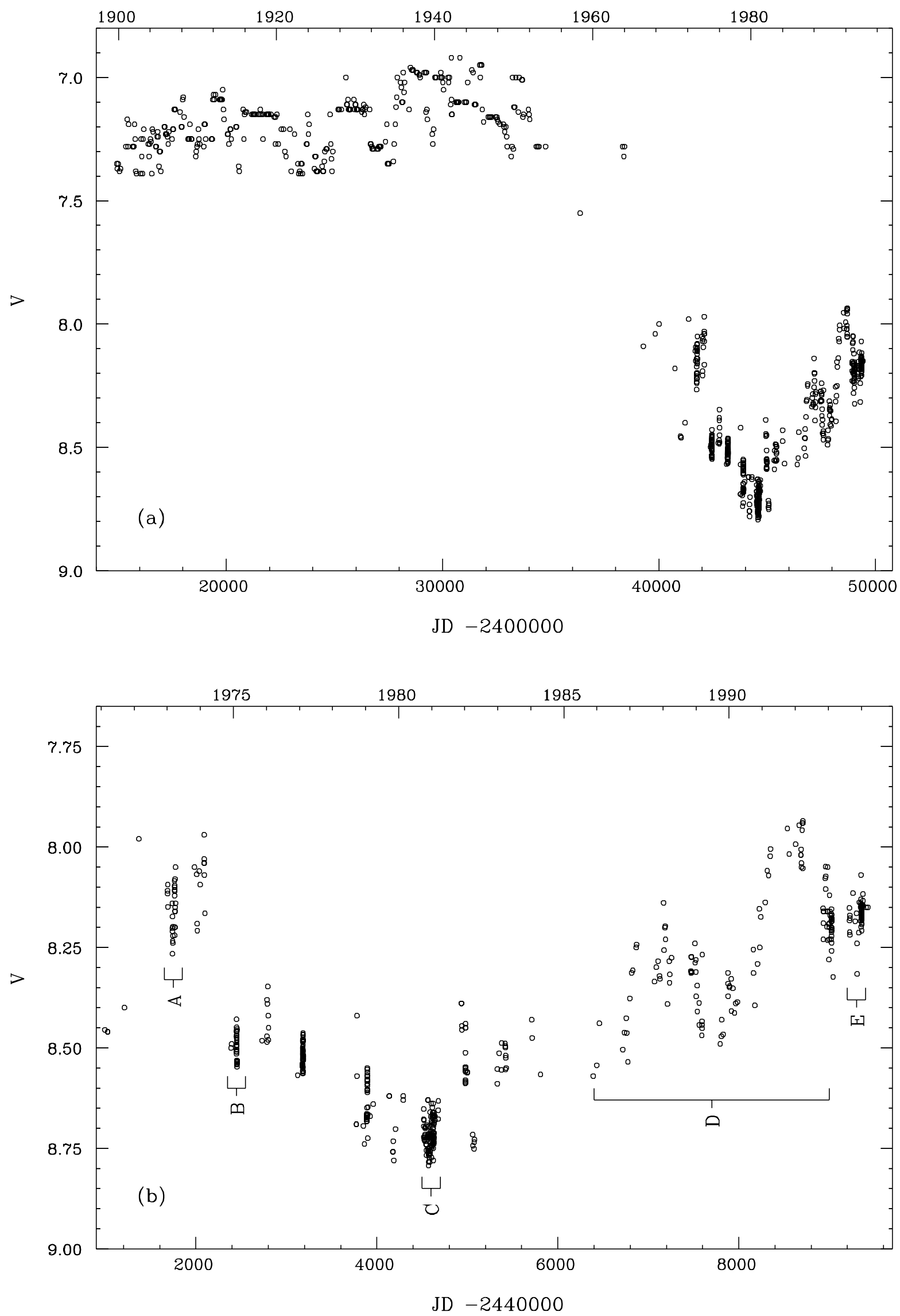

Fig. 1. Light curve of HD 45677. a) Using photographic and photoelectric data from 1899 to 1994. b) Using photoelectric data from 1971 to 1994. The labels A - E denote the different datasets discussed in Sects. 3.2 and 3.3 
Table 1. Log of published and newly taken optical spectroscopic observations of HD 45677

\begin{tabular}{|c|c|c|c|c|c|c|c|}
\hline No. & Reference & Date & Range $(\AA)$ & Dispersion & Remarks & & \\
\hline $1923-1927$ & Merrill 1928 & $1923-1928$ & Blue & Medium & Set of 9 plates & & \\
\hline $1932-1947$ & Merrill 1952 & $1932-1947$ & Blue & Medium & Set of 11 plates & & \\
\hline $1964-1971$ & Swings \& Allen 1971 & $1964-1971$ & Blue & Medium & Set of 21 plates & & \\
\hline 1966 & Burnichon et al. 1967 & $1964 / 1966$ & $3000-4900$ & Medium & & & \\
\hline $1971 \mathrm{a}$ & Ciatti et al. 1974 & $28 \quad 101971$ & Visual & Medium & & & \\
\hline $1971 \mathrm{~b}$ & Ciatti et al. 1974 & 04121971 & Red & Low & & & \\
\hline 1973 & Ciatti et al. 1974 & 28081973 & Red & Low & & & \\
\hline 1974 & Swings 1974 & -021974 & Blue & & & & \\
\hline $1974 / 1992$ & Jaschek et al. 1992 & Several & $3400-102500$ & Medium & Several types of spectra & & \\
\hline 1979 & Swings et al. 1980 & $1977 / 1979$ & $\mathrm{H} \gamma$ & High & Two sets of spectra & & \\
\hline $1988 \mathrm{a}$ & McGregor et al. 1988 & & NIR & Low & & & \\
\hline $1988 \mathrm{~b}$ & Doazan et al. 1991 & 24101988 & $\mathrm{H} \beta$ & High & & & \\
\hline $1989 \mathrm{a}$ & Halbedel 1989 & 20031987 & $\mathrm{H} \alpha$ & Medium & & & \\
\hline $1989 \mathrm{~b}$ & Jaschek et al. 1992 & 15111989 & Red & Medium & & & \\
\hline 1990 & Jaschek et al. 1993 & 07011990 & $7570-7980$ & Medium & & & \\
\hline \multicolumn{8}{|c|}{ New spectra: } \\
\hline No. & JD -2440000 & Date & $\mathrm{UT}$ & Exp. time & Range & Resolution & Remarks \\
\hline $1992 \mathrm{a}$ & 8698.6558 & 18031992 & $03: 44$ & $1800 \mathrm{~s}$ & $6098-6913 \AA$ & $0.40 \AA /$ pixel & \\
\hline $1992 \mathrm{~b}$ & 8704.5970 & 23031992 & $02: 20$ & $1800 \mathrm{~s}$ & $6098-6913 \AA$ & $0.40 \AA /$ pixel & \\
\hline $1992 c$ & 8705.5817 & 24031992 & $01: 58$ & $60 \mathrm{~s}$ & $6022-8659 \AA$ & $1.29 \AA /$ pixel & \\
\hline $1992 d$ & 8962.7479 & $06 \quad 121992$ & $05: 57$ & $300 \mathrm{~s}$ & $6090-8720 \AA$ & $1.28 \AA /$ pixel & $\mathrm{H} \alpha$ saturated \\
\hline $1992 \mathrm{e}$ & 8962.7556 & 06121992 & 06:08 & $60 \mathrm{~s}$ & $6090-8720 \AA$ & $1.28 \AA /$ pixel & \\
\hline $1992 \mathrm{f}$ & 8964.7154 & $08 \quad 121992$ & $05: 13$ & $60 \mathrm{~s}$ & $4180-7860 \AA$ & $1.80 \AA /$ pixel & $\mathrm{H} \alpha$ saturated \\
\hline $1992 \mathrm{~g}$ & 8964.7236 & 08121992 & $05: 22$ & $30 \mathrm{~s}$ & $4180-7860 \AA$ & $1.80 \AA /$ pixel & \\
\hline $1993 \mathrm{a}$ & 9267.8083 & 07101993 & $07: 24$ & $1200 \mathrm{~s}$ & $\mathrm{H} \alpha$ & 50000 & $\mathrm{H} \alpha$ saturated \\
\hline $1993 \mathrm{~b}$ & 9269.8431 & 09101993 & $08: 14$ & $300 \mathrm{~s}$ & $\mathrm{H} \alpha$ & 50000 & \\
\hline $1993 c$ & 9270.8375 & 10101993 & $08: 06$ & $180 \mathrm{~s}$ & $\mathrm{H} \alpha$ & 50000 & \\
\hline $1993 d$ & 9270.8701 & 10101993 & $08: 53$ & $180 \mathrm{~s}$ & $\mathrm{H} \alpha$ & 50000 & \\
\hline $1993 \mathrm{e}$ & 9271.8229 & 11101993 & $07: 45$ & $120 \mathrm{~s}$ & $\mathrm{H} \alpha$ & 50000 & \\
\hline $1993 \mathrm{f}$ & 9272.8340 & 12101993 & 08:01 & $120 \mathrm{~s}$ & $\mathrm{H} \alpha$ & 50000 & \\
\hline $1993 \mathrm{~g}$ & 9269.8097 & 09101993 & $07: 26$ & $300 \mathrm{~s}$ & $\mathrm{NaI} D$ & 55000 & \\
\hline $1993 \mathrm{~h}$ & 9270.8076 & 10101993 & $07: 23$ & $600 \mathrm{~s}$ & $\mathrm{NaI} D$ & 55000 & \\
\hline $1993 \mathrm{i}$ & 9271.7917 & 11101993 & 07:00 & $600 \mathrm{~s}$ & $\mathrm{NaI} D$ & 55000 & \\
\hline $1993 j$ & 9272.7979 & 12101993 & 07:09 & $600 \mathrm{~s}$ & $\mathrm{NaI} D$ & 55000 & \\
\hline $1993 \mathrm{k}$ & 9273.8563 & 13101993 & $08: 33$ & $600 \mathrm{~s}$ & $\mathrm{NaI} D$ & 55000 & \\
\hline 19931 & 9274.8833 & 14101993 & 09:12 & $600 \mathrm{~s}$ & $\mathrm{Na} I D$ & 55000 & \\
\hline $1994 \mathrm{a}$ & 9363.780 & 11011994 & $06: 43$ & $600 \mathrm{~s}$ & $\mathrm{H} \alpha$ & 55000 & \\
\hline $1994 b$ & 9364.708 & 12011994 & 05:00 & $6000 \mathrm{~s}$ & $\mathrm{Ca}$ II $K$ & 55000 & \\
\hline $1994 \mathrm{c}$ & 9365.779 & 13011994 & $06: 42$ & $3000 \mathrm{~s}$ & {$[\mathrm{O} \mathrm{I}] \lambda 6300$} & 55000 & \\
\hline $1994 d$ & 9733.2592 & 16011994 & $06: 13$ & $1200 \mathrm{~s}$ & NaI $D$ & 55000 & \\
\hline
\end{tabular}

$B_{\mathrm{J}}=B_{\mathrm{G}}+0.993$

$V_{\mathrm{J}}=V_{\mathrm{G}}+0.052$.

The $(V-I)$ and $(V-R)$ colours in the Johnson system were transformed to the Cousins system using the formulae derived by Bessell (1979). Note that the Strömgren photometry of HD 45677 in Sterken et al. (1993) are not included in Table 2 because they are in fact measurements of another star. Also note that in the paper by Feinstein et al. (1976), the labels $m_{1}$ and $c_{1}$ have been interchanged in their Table 2.

The light curve for the visual magnitude in the Johnson system, further quoted as $V$, vs. the Julian date, JD, of HD 45677 is shown in Fig. 1. From this figure one can clearly identify a very deep minimum of long duration around 1981 ( JD 2444500). Furthermore, superimposed on this relatively large variation rough changes between the different groups of data points and changes within such concentrations of measurements can be seen. These variations will be analyzed separately and quoted as long, intermediate and short time scale variations, respectively.

\subsection{Long time scale variations/obscuration}

The light curve of HD 45677 plotted in Fig. 1a shows an asymmetric, long $(\Delta t \approx 44$ years $)$, and deep $\left(\Delta V \approx 1^{\mathrm{m}} \cdot 6\right)$ minimum. Although in the beginning of the monitoring, around 1899, HD 45677 is rather stable, it seems to get brighter with increasing variations, from $7^{\mathrm{m}} \cdot 3$ with $\Delta m_{\mathrm{pg}}$ 0.2 at 1900 up to $7^{\mathrm{m}} \cdot 0$ with $\Delta m_{\mathrm{pg}} 0^{\mathrm{m}} \cdot 4$ around 1940 . From this year on HD 45677 seems to faint which probably starts to become significant around 1950. This trend could be the beginning of the deep minimum. If we extrapolate linearly by connecting the minima of the data-points of the shorter time scale variations in Fig. 1a, until the mean magnitude of 7.2 from before 1950 is reached again, we find that we expect that HD 45677 will remain increasing in brightness until about the year 2020. We believe therefore that Fig. $1 \mathrm{~b}$ only shows a part, including the minimum, of a variation in brightness on a time scale longer, $\sim 70$ years, than the one covered in our light curve.

From the photoelectric measurements taken between 1971 and 1993, connected by linear interpolation to the 


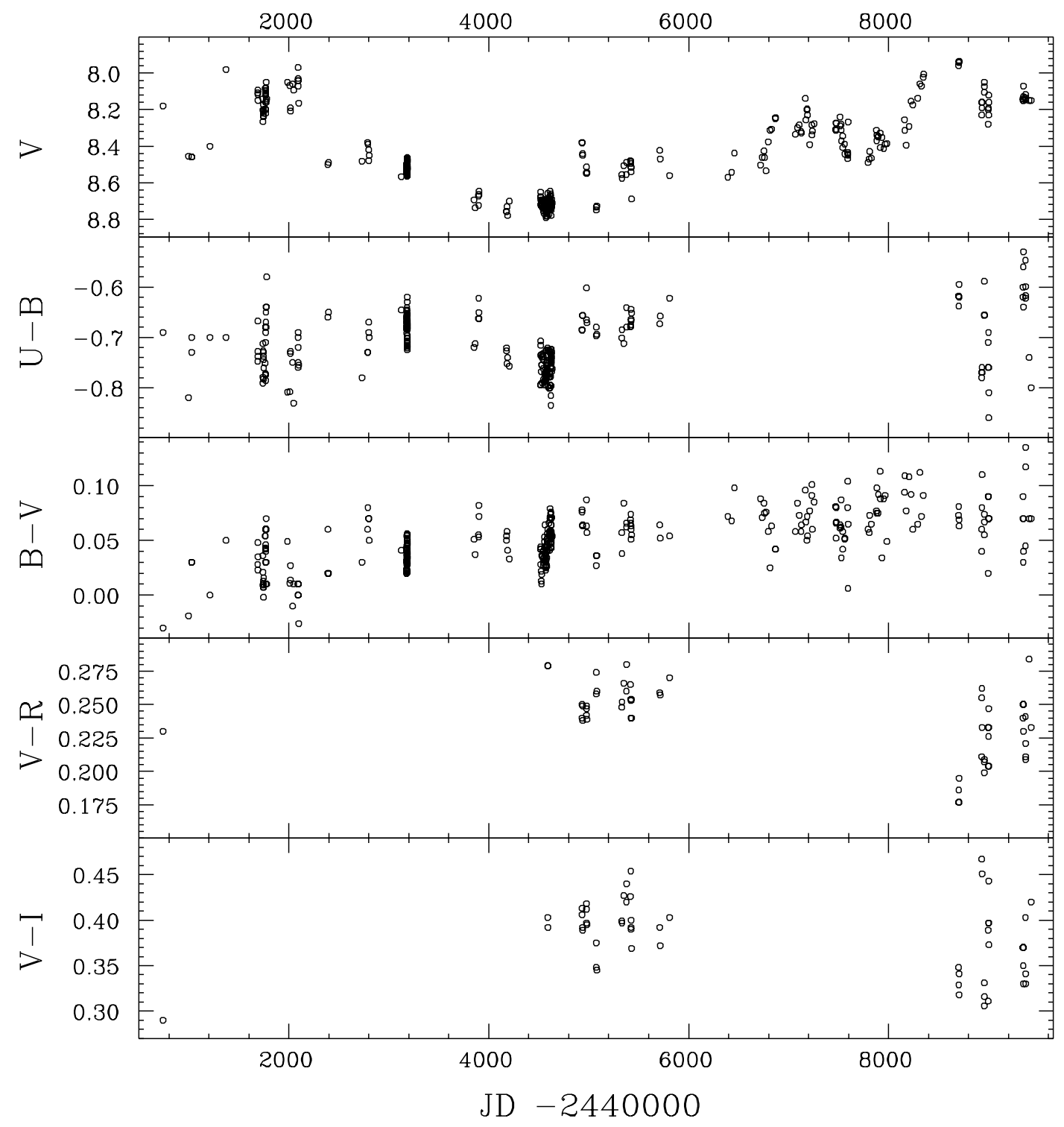

Fig. 2. Photometry of HD 45677 in the Johnson/Cousins system from 1971 to 1994

photographic magnitudes from before, the slope of the decreasing brightness is seen steeper than the branch of increasing brightness which starts at the turning point at $\sim 1981$. By the extrapolation to 2020, the speeds of the brightness changes are determined to be 0.05 year $^{-1}$ and $00^{\mathrm{m}} 04$ year $^{-1}$ for the decreasing and increasing branches, respectively. The difference, which is significant on this time scale, means that the mechanism responsible for the increasing branch is not simply a reversed effect of the mechanism causing the decrease in brightness.

Changes in photometric colour indices are measurable, but relatively small compared to the ones in $V$. Figure 2 show the light curves in both visual magnitude and colours in the Johnson/Cousins photometric system over the last 20 years. From these figures it is easily seen that, super- imposed on variation on smaller time scales, the $U-B$ and $B-V$ colours have shown a trend of getting redder during the last 20 years by about 0.1 , during the phase of increasing brightness. The scatter in the data of these colours is too large to follow this trend during the obscuration phase as well, but an indication of this behaviour can be seen in the $B-V$ colour.

The opposite colouring, getting bluer, is seen for $V-R$ and $V-I$ during the period of increasing brightness.

\subsection{Short time scale variations/flickering}

To understand the way the intermediate variations are distributed it is better first to explain the short time scale variations, i.e. to study whether they are caused by obscurations or brightenings. Short time scale 
Table 2. Visual photometric data of HD 45677 from the literature

\begin{tabular}{lll}
\hline $\mathrm{JD}-2400000$ & Photometric system & Reference \\
\hline $14943-38409$ & Photographic & Swings \& Swings (1972) \\
$\approx 36360$ & $U B V$ & Mendoza (1958) \\
$\approx 39280$ & $u v b y \beta$ & Cameron (1966) \\
$\approx 39830$ & $U B V$ & Haupt \& Schroll (1974) \\
$\approx 40010$ & $U B V(R)_{\mathrm{J}}$ & Low et al. (1970) \\
$\approx 40740$ & $U B V(R I)_{\mathrm{J}}$ & Low et al. (1970) \\
$\approx 41025$ & $U B V(R I)_{\mathrm{J}}$ & Swings \& Allen (1971) \\
$\approx 41025$ & $U B V$ & Penston (1973) \\
$41369-42802$ & $U B V+u v b y$ & Feinstein et al. (1976) \\
$40994-44633$ & $\mathrm{Geneva} U B_{1} B B_{2} V_{1} V G$ & Gilbert (1994) \\
$42784-45808$ & $U B V(R I)_{\mathrm{C}}+u v b y \beta$ & Kilkenny et al. (1985) \\
$43124-43902$ & $U B V+u v b y$ & de Winter et al. (1996) \\
$44596-44633$ & Walraven $W U L B V$ & de Winter et al. (1996) \\
$46391-47595$ & $B V$ & Halbedel (1989) \\
$47793-48350$ & $B V$ & Halbedel (1991) \\
$43773-48978$ & $\mathrm{IUE} V(\mathrm{FES})$ & Pérez (1994) \\
$48500-49200$ & $B V$ & Sitko et al. (1994) \\
$48932-48997$ & $U B V(R I)_{\mathrm{J}}$ & Bergner et al. (1995) \\
$44589-49372$ & $U B V(R I)_{\mathrm{C}}$ & de Winter et al. (1996) \\
$49022-49359$ & $u v b y \beta$ & de Winter et al. (1996) \\
\hline
\end{tabular}

variations are seen in the lightcurve as dense groups of data points. The typical time scales vary from several days to several hours, depending on the monitoring programmes. They can be easily studied by constructing light curves and colour-magnitude diagrams over several short periods during the last twenty years. Pérez et al. (1993) suggested the presence of short time scale "pulse-like", or "flickering", type of variations, characteristic of instabilities in accretion flows.

We examined light curves and colour-magnitude diagrams of several datasets obtained within several days, which are denoted in Fig. $1 \mathrm{~b}$ as A-E. $U B V$ and Strömgren data of Feinstein et al. (1976), sets A and B respectively, are shown in Fig. 3. As can be seen from this figure, HD 45677 shows irregular minima and maxima of which the time scale seems to be up to days, see plot B1 of Fig. 3. These "pulse-like" variations from night to night, and their colours clearly indicate a dependence on magnitude: the blue colours, $U-B, u-v$ and $v-b$ are getting redder as the magnitude decreases but then colours like $B-V$ and $b-y$ become bluer. A better example of the "pulse-like" behaviour and their colour effects are seen in the same figure for the transformed Geneva colours of Gilbert (1994), set C, and the $B V$ observations of Halbedel (1989), set D (see next section). At decreasing brightness the $U-B$ colour of sets $\mathrm{A}$ and $\mathrm{C}$ gets redder while $B-V$ becomes bluer again. The amount of the getting redder seems to increase slightly towards the blue, i.e. most strongest in $u-v$. Even in the colours $U-B$ and $v-b$ the getting bluer is significant higher than the getting redder in the $b-y$ and $B-V$ colours. The Walraven data in Table 1 of Paper I confirms this behaviour.

\subsection{Intermediate time scale variations/semi periodic}

The intermediate time scale variations, with typical time scales of a few years, were studied and found to be semiperiodic by Halbedel (1989). However, the monitoring from 1899 to 1969 by Swings \& Swings (1972) indicated no presence of clear periodicity on such time scales.

A clear representation of these changes, in the photoelectric data, are only given beyond JD $\sim 2446000$. Before this, HD 45677 was not monitored sufficiently regular. The empty intervals in the lightcurve (Fig. 1b) are the reason for the difficulties to explain these variations by Halbedel (1989) and Feinstein et al. (1976). Producing colour-magnitude diagrams for the intermediate time scale variations is not easy because of the different photometric systems used for the measurements. However, using the $B V$ data from Halbedel $(1989,1991)$, Sitko et al. (1994) and from Paper I, which extend over several similar years, a slight relation is seen between $B-V$ and $V$ (Fig. 3). Superimposed on the short time scale variations and their colour dependencies a reddening is seen with decreasing brightness. Because of the lack of other colours measured over such time intervals their effects are not known.

\section{Analysis of the spectroscopic behaviour}

As we have seen dramatic changes in the photometry of HD 45677 on different time scales one could expect to see such changes in the spectroscopic behaviour as well. Indeed, spectral variations have been observed for the lines of Mg II, He I and slightly in Ca II (Swings et al. 1980). Because of the high radial velocities measured for the He I and $\mathrm{Mg}$ II lines, $150-200 \mathrm{~km} \mathrm{~s}^{-1}$, those lines are thought to be formed close to the stellar surface. For the $\mathrm{H} \delta$ and $\mathrm{H} \gamma$ lines more dramatic variations are seen as well, most significantly in the violet parts of the profiles and on time scales ranging from hours up to days (Merrill 1952; Swings et al. 1980). Similar variations in the $\mathrm{H} \gamma$ profiles were already reported on a longer time scale on plates taken between 1923 and 1947 (Swings \& Struve 1943; Merrill 1952). Although Swings \& Allen (1971) did not report any correlations of line variations with detected photometric changes, we can give a more exact view at the moment since more spectroscopic observations overlap with the different time scales of the photometric variations.

To study the long time scale variations we use as a starting point the spectral descriptions of Merrill (1928) and of Swings et al. (1980). We have searched for additional spectra which are listed in Table 1. This summary is certainly not complete as probably many plate and CCD spectra are reported but not discussed, e.g. Jaschek et al. (1992), or unpublished, e.g. Swings (1995). Several of such spectra, as well as many others listed in Table 1, will be discussed in detail by Israelian et al. (1996). We refer to the spectra in the following sections by their number, the year of observation, as listed in Col. 1 of Table 1. Most 

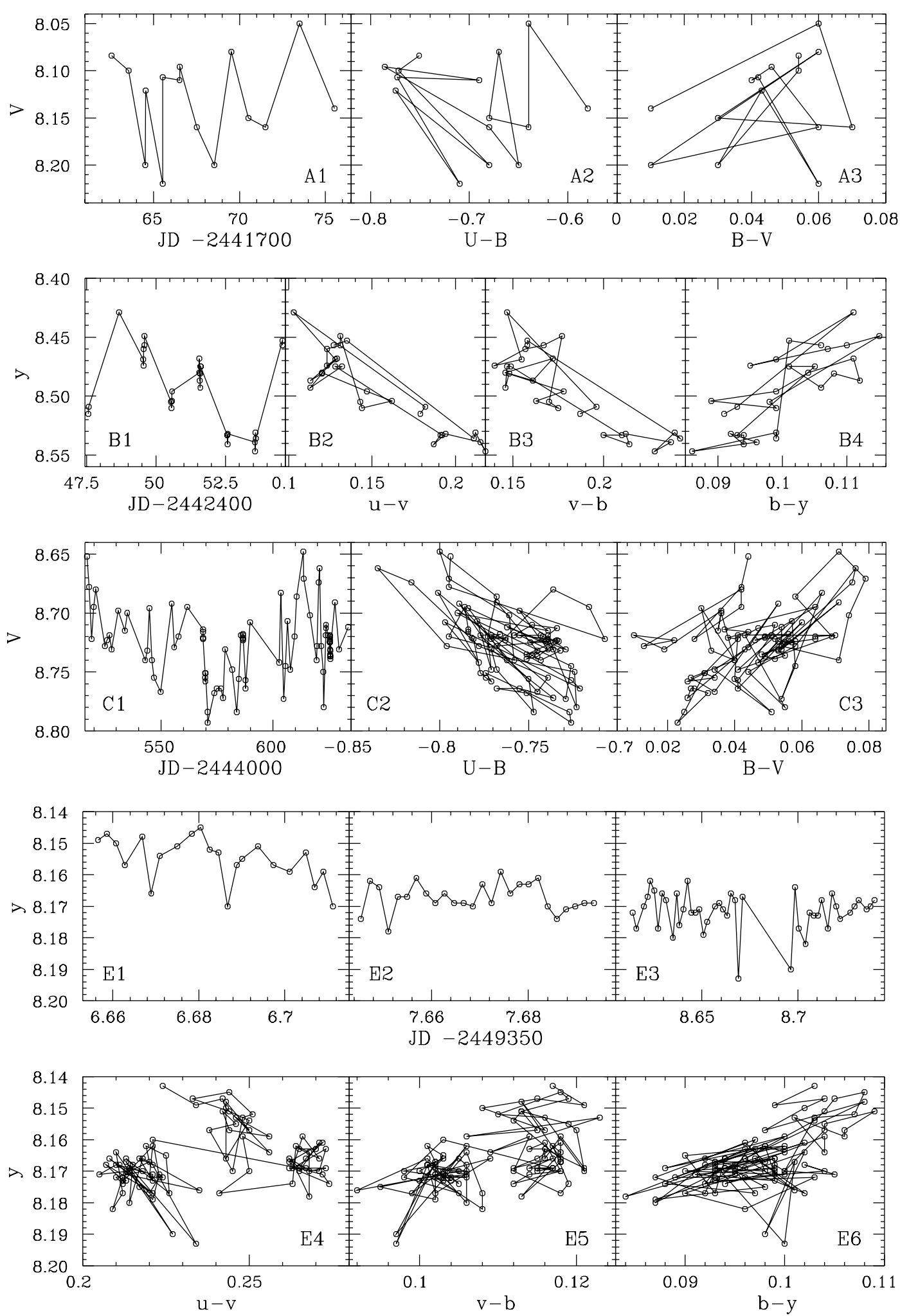

Fig. 3. The short time scale variations expressed in light curves and in colour-magnitude diagrams for the datasets taken in 1972, set A ( $U B V$ data of Feinstein et al. 1976); 1975, set B (uvby data of Feinstein et al. 1976); 1981, set C (Gilbert 1994) and 1993, set E (Table 2) 

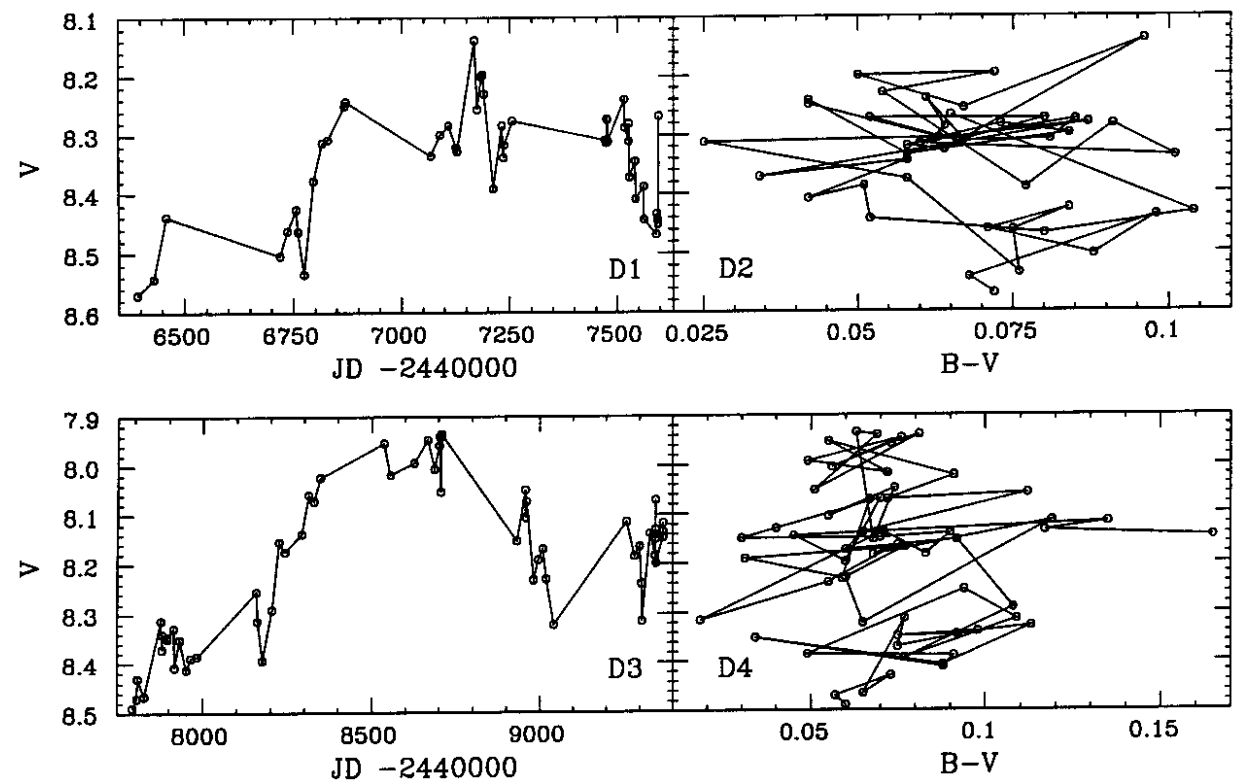

Fig. 3. continued. The intermediate time scale variations expressed in light curves and in colour-magnitude diagrams for the datasets taken between 1986 and 1993, set D, by Halbedel (1989 and 1991), Sitko et al. (1994) and from Paper I

historical known spectra are of low resolution. It is therefore only possible to make use of our own low and intermediate resolution spectra for these purposes. As our low resolution spectra are quite similar we have only presented the 1992e and 1992g in Fig. 4, together with the line identifications which will be discussed. For a similar reason the 1992b intermediate resolution spectrum is not shown in Fig. 5. However, from the presented $\mathrm{H} \alpha$ profiles clear changes are seen.

Investigating such short time scale variations, as also known from the literature, we use our monitoring data. We have obtained time series with a duration of 6 days by high resolution spectroscopy in two wavelength regions. Besides spectra centered on the $\mathrm{H} \alpha$ laboratory wavelength, with a resolving power of 50000 , we have monitored the Na I $D$ lines, with a resolving power of 55000 . The choice for this line is inspired by the clear detection of accreting cool material in the case of the Herbig Ae star UX Ori (Grinin et al. 1994). Furthermore, our setup includes the He I line at $5876 \AA$ and the [Fe II] line at $5870 \AA$. The Na I $D$ and $\mathrm{H} \alpha$ time series were taken in October 1993. One other spectrum, in both regions, was obtained in January 1995. The spectra are plotted in Figs. 9a and 10a.

There is no spectral data that would illustrate changes on intermediate time scales in either low or high resolution. This will therefore not be discussed.

\subsection{Long time scale variations}

The existence of an extended gaseous shell of considerable density was first inferred from the appearance of nebu- lar lines of [O I $]$ at $6300 \AA$ and $6363 \AA$ and of $[\mathrm{S} \mathrm{II}]$ at $4068 \AA$ (Merrill \& Burwell 1933; Swings \& Allen 1971).

Contrary to spectroscopic variations as mentioned above, the forbidden lines, like [FeII], [O I], [N II], are stable; no clear changes are seen since the first detections. Probably those lines are formed in an extended region far away from the central star, see Fig. 13 of Swings (1973). The red [S II] lines were not detected and the [N II] lines very weak. As reported by Swings (1973), the Fe II lines are double peaked and more dispersed than the [Fe II] lines.

Because of the wide variety of the spectroscopic data in wavelength coverage and resolution we will discuss them by each group separately. In some cases a more detailed description will be given by Israelian et al. (1996).

\subsubsection{Visual: Low and intermediate resolution spectra}

In the 1971 and 1992 spectra, $\mathrm{H} \alpha$ is seen in emission accompanied at the red side by the $[\mathrm{NII}]$ line. In the intermediate resolution 1992a and b spectra $\mathrm{H} \alpha$ is also accompanied at the blue side by the weaker [N II] line of the same multiplet. Both are in emission. It is also seen in most other spectra, like in 1989 , in which $\mathrm{H} \alpha$ shows self-absorption. The velocities and line profiles measured in the 1989 spectrum are similar to those reported before, e.g. Merrill (1952). However, the $\mathrm{H} \alpha$ profile is very complex. We will discuss this further in Sect. 4.2.2.

In the 1992c-e spectra, as in the previous ones, the O I lines at $7774 \AA$ and $8446 \AA$ are very strong. The [S II] lines, which were not detected before, are weakly in emission (spectra 1992a and b). No differences can be seen for the Fe II lines in the 1992 spectra and those observed 


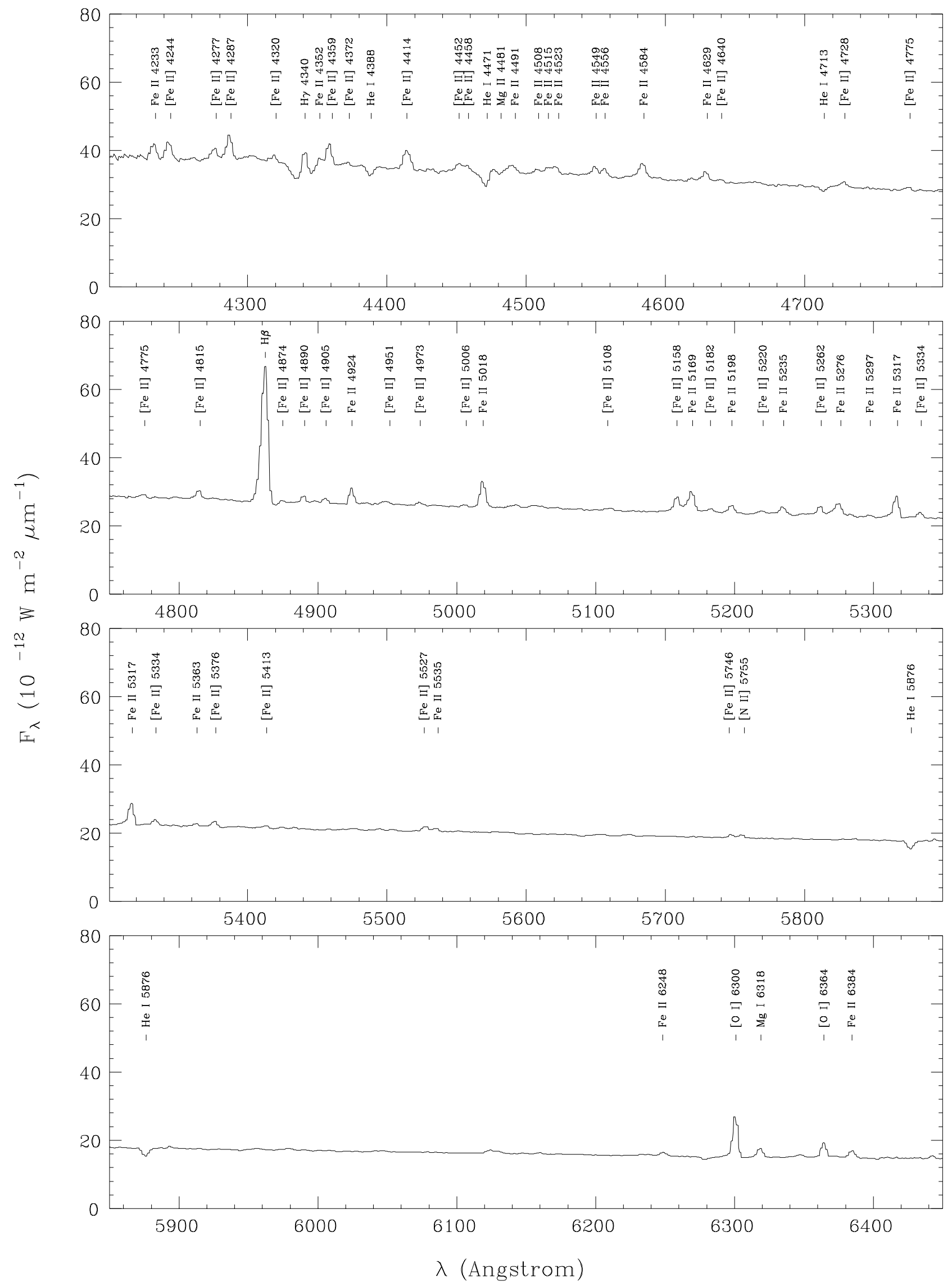

Fig. 4. December 1992 low-resolution spectra of HD 45677 with the most prominent lines identified 


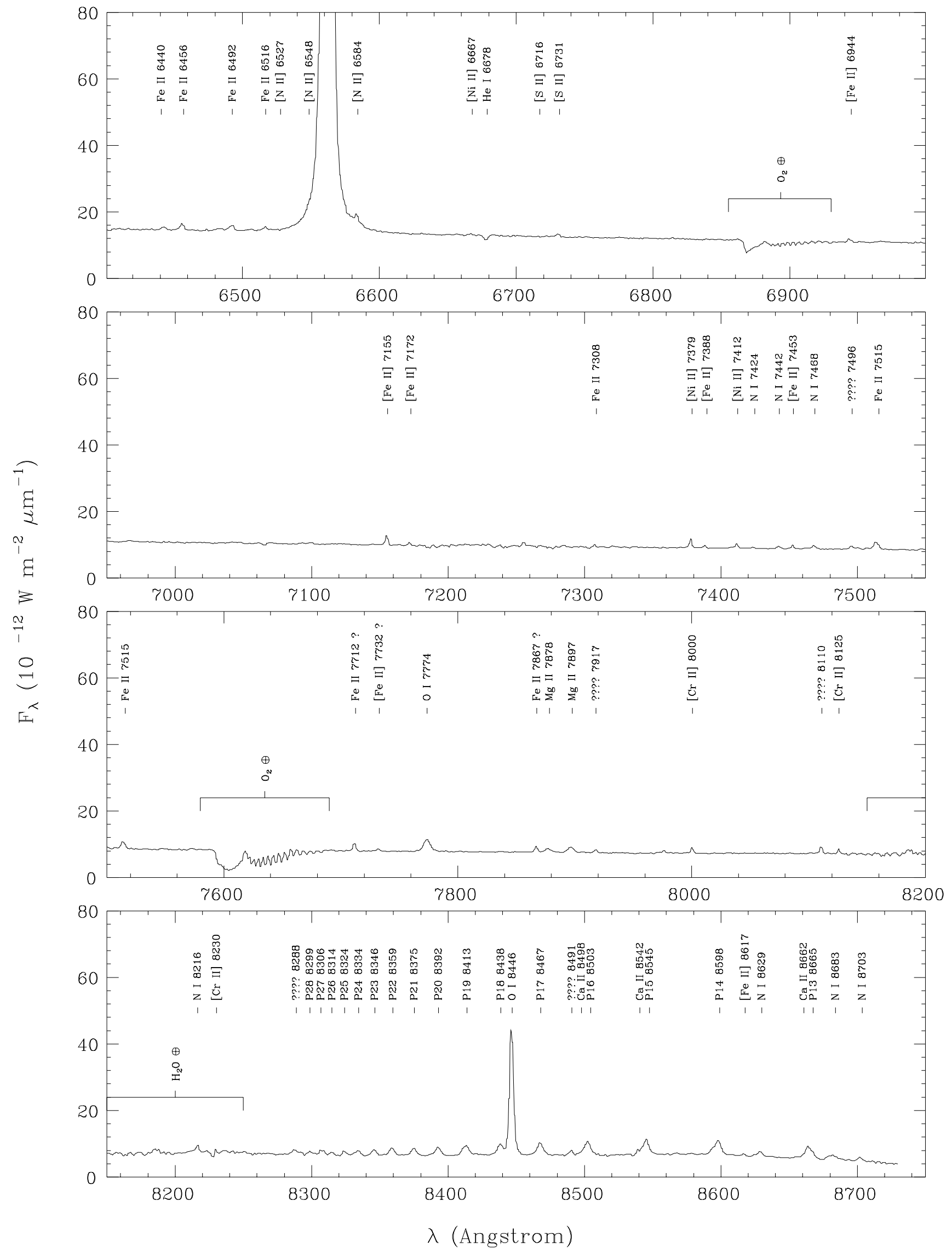

Fig. 4. continued 
Table 3. Identified emission lines in the low- and intermediate-resolution spectra of HD 45677, Figs. 4 and 5. Uncertain identifications are given between brackets

\begin{tabular}{|c|c|c|}
\hline Element & Multiplets & $n$ \\
\hline $\mathrm{H} \mathrm{i}$ & $1,9,10,11,12,(13)$ & $3: \alpha \beta \gamma, 2,5,5,4,(1)$ \\
\hline $\mathrm{Ni}$ & $1,2,3$ & $3,1,2(1)$ \\
\hline $\mathrm{O}$ i & 1,4 & 3,3 \\
\hline $\mathrm{Mg}$ i & $2,14,23$ & $1,2,2$ \\
\hline Mg ii & 8,16 & 2,1 \\
\hline Ca ii & 2 & $1(2)$ \\
\hline Fe ii & $\begin{array}{l}27,37,38,40,42,(46) \\
48,49,55,74, ?\end{array}$ & $\begin{array}{l}2(1), 5(1), 4,2,3,(2), \\
2(2), 4,1,2(4), 7\end{array}$ \\
\hline$[\mathrm{Ni}]$ & 1 & 2 \\
\hline$[\mathrm{N} \mathrm{ii}]$ & 1,3 & $2(1), 1$ \\
\hline$[\mathrm{O} \mathrm{i}]$ & 1 & 2 \\
\hline [S ii $]$ & 2 & 2 \\
\hline [Cr ii] & 1 & 4 \\
\hline$[\mathrm{Fe}$ ii $]$ & $\begin{array}{l}6,7,13,14,15,17 \\
18,19,20,21,34,43\end{array}$ & $\begin{array}{l}2,4(1), 1,3,2,4 \\
2(1), 4(1), 8(1), 3,1,1\end{array}$ \\
\hline$[\mathrm{Ni}$ ii $]$ & 2 & 2 \\
\hline
\end{tabular}

before. The $\mathrm{H} \gamma$ and other blue hydrogen line profiles confirm the detections given by Swings (1973) and Swings et al. (1980). From 1964 to 1966 emission components appeared in the blue hydrogen lines, from H10 up to H5 (= $\mathrm{H} \epsilon)$. In $1979 \mathrm{H} \gamma$ shows central emission. In 1974, Swings (1974) reported that $\mathrm{H} \gamma$ exhibits a very strong $\mathrm{P}$ Cygni profile. In 1966 no emission is seen. Furthermore, the known strong iron emission lines were observed double peaked, separated by $\Delta v \approx 30 \mathrm{~km} \mathrm{~s}^{-1}$, being the same value as reported above. In the 1992a and b spectra $\mathrm{H} \gamma$ is seen in emission with a central emission embedded in a photospheric absorption line, as the profile was observed in the 1971 spectra. In the 1992 intermediate resolution spectra we also note that the FeII lines are relatively broad, when compared to typical nebular lines as [N $\mathrm{II}$, $[\mathrm{S} I \mathrm{I}]$ and $[\mathrm{O} \mathrm{I}]$ in the same spectra. The Fe II lines also hide traces of double peaked profiles with a separation of about $40-60 \mathrm{~km} \mathrm{~s}^{-1}$, which is somewhat higher than in the spectra taken before the photometric minimum. Although the Fe II lines are relatively broad and the resolution of the 1992 spectra are too low to determine good velocities, most identified lines seem to be centered on a velocity of about $20 \mathrm{~km} \mathrm{~s}^{-1}$.

The properties of the described spectra are similar to those observed and discussed by Swings (1973) and Swings et al. (1980). Even the short time scale variations reported in these papers are seen in the 1992 intermediate resolution spectra. We have compared both of the spectra, 1992a and 1992b. The difference spectrum (not shown here) clearly indicates significant changes in the $\mathrm{H} \alpha$ line while all other lines seem to be stable. In the 1992b spectrum we clearly have a broad extra absorption at the blue side of the $\mathrm{H} \alpha$ spectrum, as well as two small absorption components at +4 and $-60 \mathrm{~km} \mathrm{~s}^{-1}$. In the next section we will discuss the $\mathrm{H} \alpha$ profile in more detail.

\subsubsection{Visual: high resolution spectra}

High resolution spectra of HD 45677 are rare. A comparison of our data with other spectra, besides the hydrogen lines, is therefore not possible. However, we will discuss here the spectra mentioned in Table 1 of Ca II $K$ (Fig. 6) and [O I] $\lambda 6300$ (Fig. 7) including the $\mathrm{Mg}$ I $6318.23 / .75 \AA$ doublet (Fig. 8). The other high resolution spectra will be discussed in the next section.

A $\mathrm{H} \beta$ spectrum from Doazan et al. (1991) shows a strong double peaked emission line with the red component much stronger than the violet peak similar to the 1989a spectrum and the ones reported before (e.g. Merrill 1928). However, the peak separation in the $\mathrm{H} \beta$ spectra by Doazan et al. (1991) and by Merrill (1928) is much bigger (150 and $170 \mathrm{~km} \mathrm{~s}^{-1}$, respectively) than in all the $\mathrm{H} \alpha$ spectra $\left(\approx 75 \mathrm{~km} \mathrm{~s}^{-1}\right)$. This difference supports our interpretation of the double-peaked structure of the hydrogen lines as being due to self-absorption rather than being due to two separate regions of hydrogen emission. For our 1993 $\mathrm{H} \alpha$ spectra the two peaks differ less in strength than in the 1988b H $\beta$ spectrum, while in the $1992 \mathrm{a}$ and b $\mathrm{H} \alpha$ spectra we even have $V / R \approx 1$. This indicates a strong variability of the hydrogen lines as noted previously by Swings et al. (1980).

The CaII $K$ line is seen with the absorption center at $20 \mathrm{~km} \mathrm{~s}^{-1}$. At the blue and red side of this narrow line (the maximum velocity dispersion, at the continuum level, is less than $50 \mathrm{~km} \mathrm{~s}^{-1}$ ) weak emission is seen. This profile is identical to the ones from 1932 to 1947 (Merrill 1952) and similar to the one mentioned by Swings (1973). The [O I] $\lambda 6300$ and the $\mathrm{Mg}$ I doublet are in emission with the central peak at about $20 \mathrm{~km} \mathrm{~s}^{-1}$. Although the velocity dispersion of the [O I] line is higher, about $90 \mathrm{~km} \mathrm{~s}^{-1}$, than the Ca II line, it is much lower than the $250 \mathrm{~km} \mathrm{~s}^{-1}$ of the $\mathrm{Mg}$ I doublet. Note also that the latter is asymmetric with extra emission at the blue side of its profile extending to $150 \mathrm{~km} \mathrm{~s}^{-1}$, whereas in the red part the velocity goes up to about $100 \mathrm{~km} \mathrm{~s}^{-1}$.

From the velocities we conclude that the $\mathrm{Mg}$ I line is formed closer to the central star than the $[\mathrm{O}$ I] $\lambda 6300$, which must be formed in an extended low density region. Although Mg I will be destroyed close to the central star there must be some outflow of this material. Also the emission in the Ca II resonance line shows the presence of some cool material. When distributed in a disk this material must be very dense as seen by the strong self-absorption line.

\subsubsection{Red spectra}

In comparing the red spectra of 1971b and 1973, although the $1971 \mathrm{~b}$ spectrum is of poor quality, we see in the $1971 \mathrm{~b}$ spectrum He I $6678 \AA$ in emission but not in the 1973 spectrum. In 1973 Fe II $7712 \AA$ and O I $7774 \AA$ are in emission but in $1971 \mathrm{~b}$ weak or probably not. In the red part of 

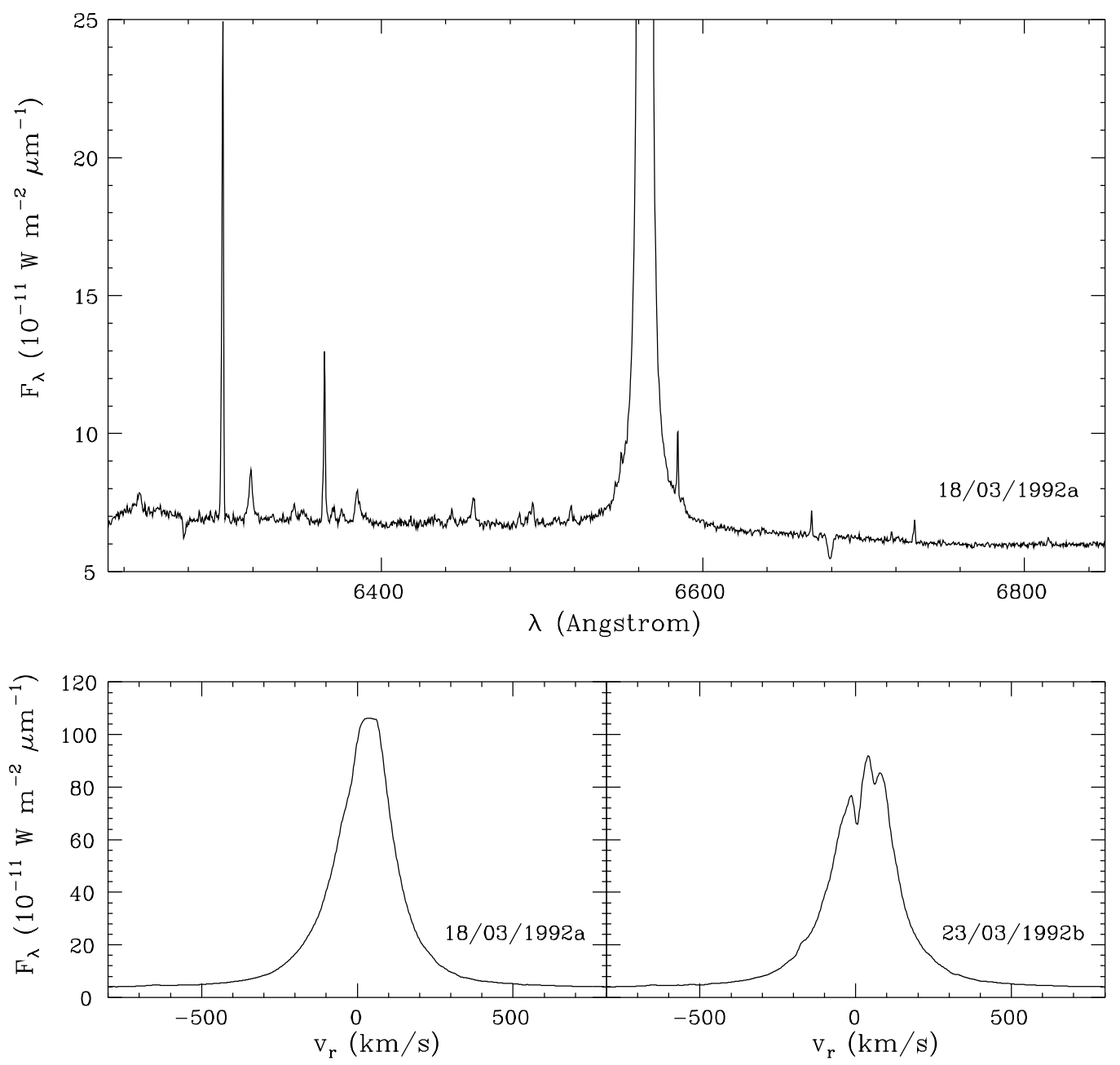

Fig. 5. May 1992 intermediate-resolution spectra of HD 45677. The full wavelength range of the 1992a spectrum is shown, the 1992b spectrum is very similar and therefore not included. However, line profiles can be very different as shown in the lower plots for $\mathrm{H} \alpha$

1992c and in 1992d the FeII $7712 \AA$, [FeII] $7732 \AA$ and O I $7774 \AA$ are seen in emission, similar to the 1973 situation. In 1993 these lines are also visible in emission, only the O I line was weaker than it was in $1992 \mathrm{~d}$. In the $1989 \mathrm{~b}$ spectrum the O I $8446 \AA$ is strong in emission, also the Paschen lines and many FeII lines are in emission, very similar to the 1973 situation. The same wavelength region of the $1992 \mathrm{~d}$ spectrum looks exactly like in the previous ones, with the exception of the one detecting the decreasing strength of O I $7774 \AA$.

\subsubsection{NIR spectra}

The 1988 spectra look exactly as the ones of HD 87643 and $\mathrm{CD}-42^{\circ} 11721$, two other well known B[e]-stars. Only Hlines are in emission, no other features are seen. No other corresponding spectra are available for comparison.

\subsection{Short time scale variations}

The high resolution sodium and $\mathrm{H} \alpha$ line profiles are presented in Fig. 9a and Fig. 10a, respectively. Their characteristics will be presented separately. A more qualitatively modeling on these spectra will be presented in another paper (Israelian et al. 1996).

\subsubsection{The sodium spectra}

It is striking to see that in all spectra the Na I $D$ lines are significantly in emission with a strong absorption core at $20 \mathrm{~km} \mathrm{~s}^{-1}$, similar to the observed Ca II $K$ line. The emission components of the $D_{1}$ lines is somewhat stronger than the $D_{2}$ line, as usual, but combined with the relatively strong self-absorption component the $D_{2}$ profile looks somewhat different. The interstellar component is not visible and is probably blended in the broad circumstellar absorption feature. 
Between the spectra taken in 1993 and the one of 1995, no dramatic changes are seen, although the strong telluric waterlines could give a wrong impression. However, comparing the time series spectra to each other, using the difference between a spectrum and the one of 09/10/1993, it is clear that day by day variations are significant in both the Na I $D$ and the He I lines (Fig. $9 \mathrm{~b}$ ). Note the changes of the emission strength at the blue sides of the Na I $D$ lines. However, the wings of the red sides of these lines are remarkably stable as they all extent up to $147 \pm 5 \mathrm{~km} \mathrm{~s}^{-1}$ for the $D_{1}$ and up to $140 \pm 5 \mathrm{~km} \mathrm{~s}^{-1}$ for the $D_{2}$ components. Similar profiles, but with more dramatic changes, are observed by Israelian et al. (1996) on a somewhat longer time scale.

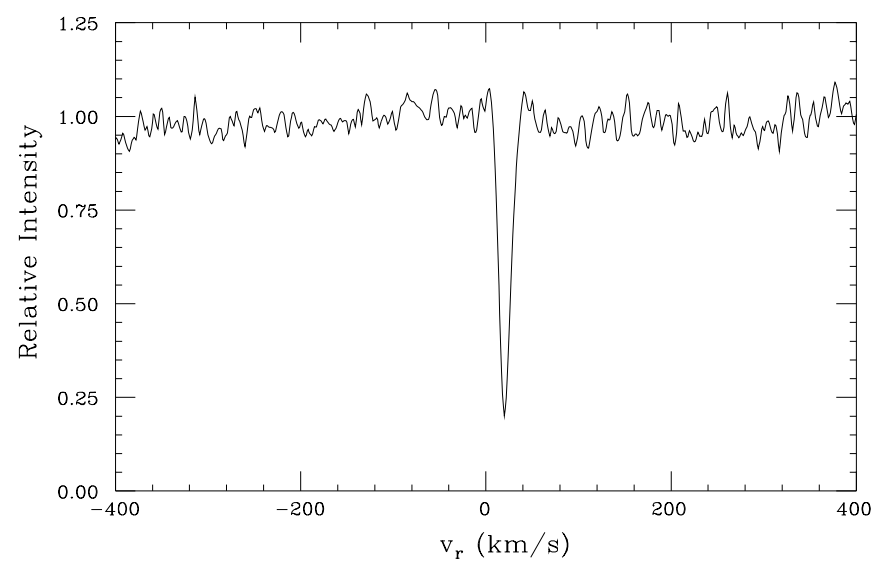

Fig. 6. High resolution CaII $K$ spectrum of HD 45677, rebinned to heliocentric velocities

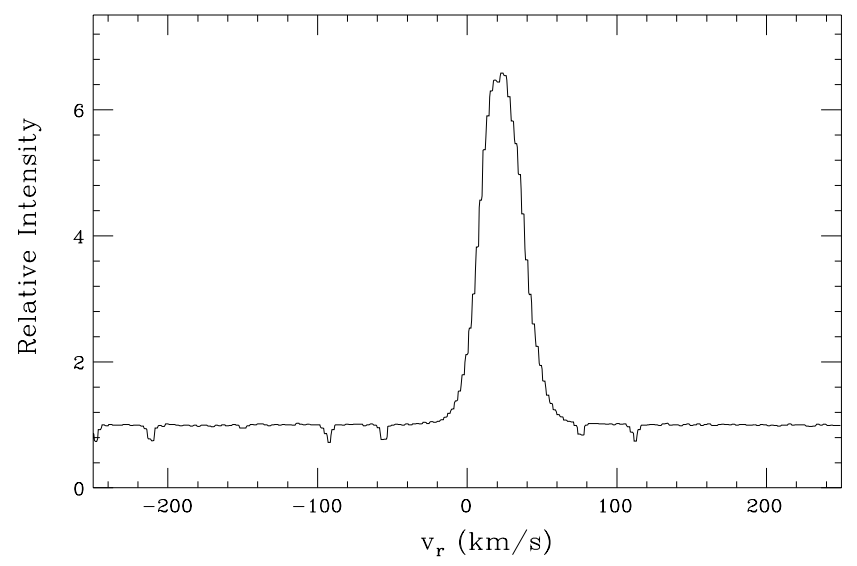

Fig. 7. High resolution [O I] $\lambda 6300$ spectrum of HD 45677, rebinned to heliocentric velocities

In the spectra of 13 and 14/10/1993 the blue emission wings are quite strong and extend up to -99 $\pm 3 \mathrm{~km} \mathrm{~s}^{-1}$ and $-65 \pm 5 \mathrm{~km} \mathrm{~s}^{-1}$, respectively, while on

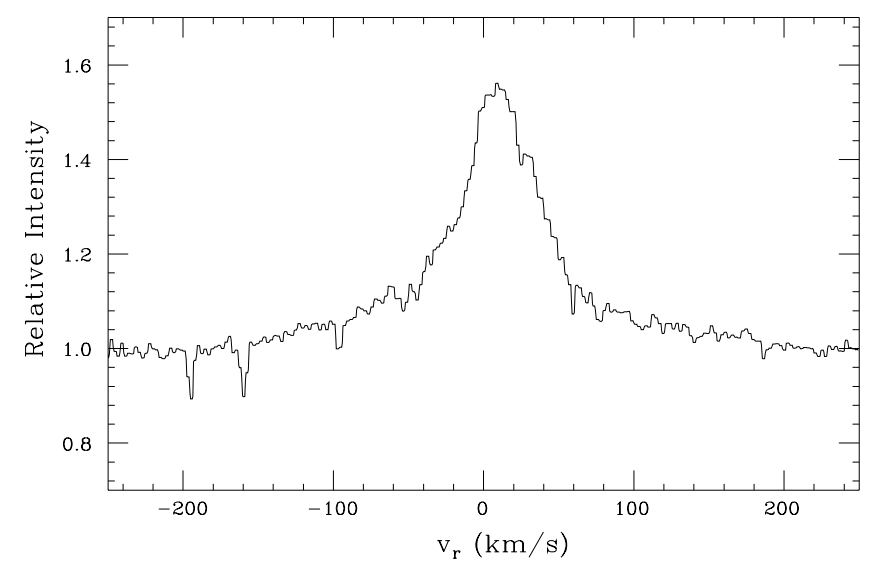

Fig. 8. High resolution spectrum of the $\mathrm{MgI} 6318.23+$ $6318.75 \AA$ doublet of $\mathrm{HD} 45677$, rebinned to heliocentric velocities

10 and 11/10/1993 these blue parts are depressed, and show even weak narrow absorption components at -18 $\pm 1 \mathrm{~km} \mathrm{~s}^{-1}$ (not to be confused with the telluric waterline as seen in the $16 / 1 / 1995$ spectrum). On 09 and $12 / 10 / 1993$ these weak lines are located at -11 $\pm 1 \mathrm{~km} \mathrm{~s}^{-1}$. An extra wide but very weak absorption is also seen on $11 / 10 / 1993$ at $-75 \pm 2 \mathrm{~km} \mathrm{~s}^{-1}$.

The blueshifted weak absorption components seem to be dynamical on a daily time scale, but can also appear for a few days, with relatively large changes in their velocities. In our time series this absorption slowly disappears and in the last two spectra, 13 and 14/10/1993, only extra emission is seen.

\subsubsection{The $\mathrm{H} \alpha$ lines}

Several $\mathrm{H} \alpha$ spectra were taken in the same nights as the Na I $D$ spectra. The one taken on $11 / 01 / 1994$ is somewhat saturated, as is the $09 / 10 / 1993$ spectrum slightly overexposed. As we noticed only small daily changes between the Na I $D$ spectra, the same seems to be the case for the quasi-simultaneously taken $\mathrm{H} \alpha$ profiles, see Fig. 10a. The $\mathrm{H} \alpha$ profiles are quite complex. A strong self-absorption component at $+11 \pm 1 \mathrm{~km} \mathrm{~s}^{-1}$ is visible in all the spectra. However, it is not located at the center of the emission profile.

We have determined the center of the emission profile by the fitting of a Gaussian to the observed profiles and to modified ones, in which the absorption components are deleted by interpolation. The results of both fitting procedures are almost identical. The emission peak seems to shift from $+37.5 \pm 1 \mathrm{~km} \mathrm{~s}^{-1}$ for the $09 / 10 / 1993$ profile to $+40.5 \pm 1 \mathrm{~km} \mathrm{~s}^{-1}$ for the $12 / 10 / 1993$ spectrum, with a daily step of $+1 \mathrm{~km} \mathrm{~s}^{-1}$. This is almost $10 \mathrm{~km} \mathrm{~s}^{-1}$ higher than the radial velocity of the $\mathrm{H} \alpha$ lines before 1928 (Merrill 1928). The velocity offset between the $\mathrm{H} \alpha$ emission and systemic velocity from +17.5 to $+20.5 \mathrm{~km} \mathrm{~s}^{-1}$ 

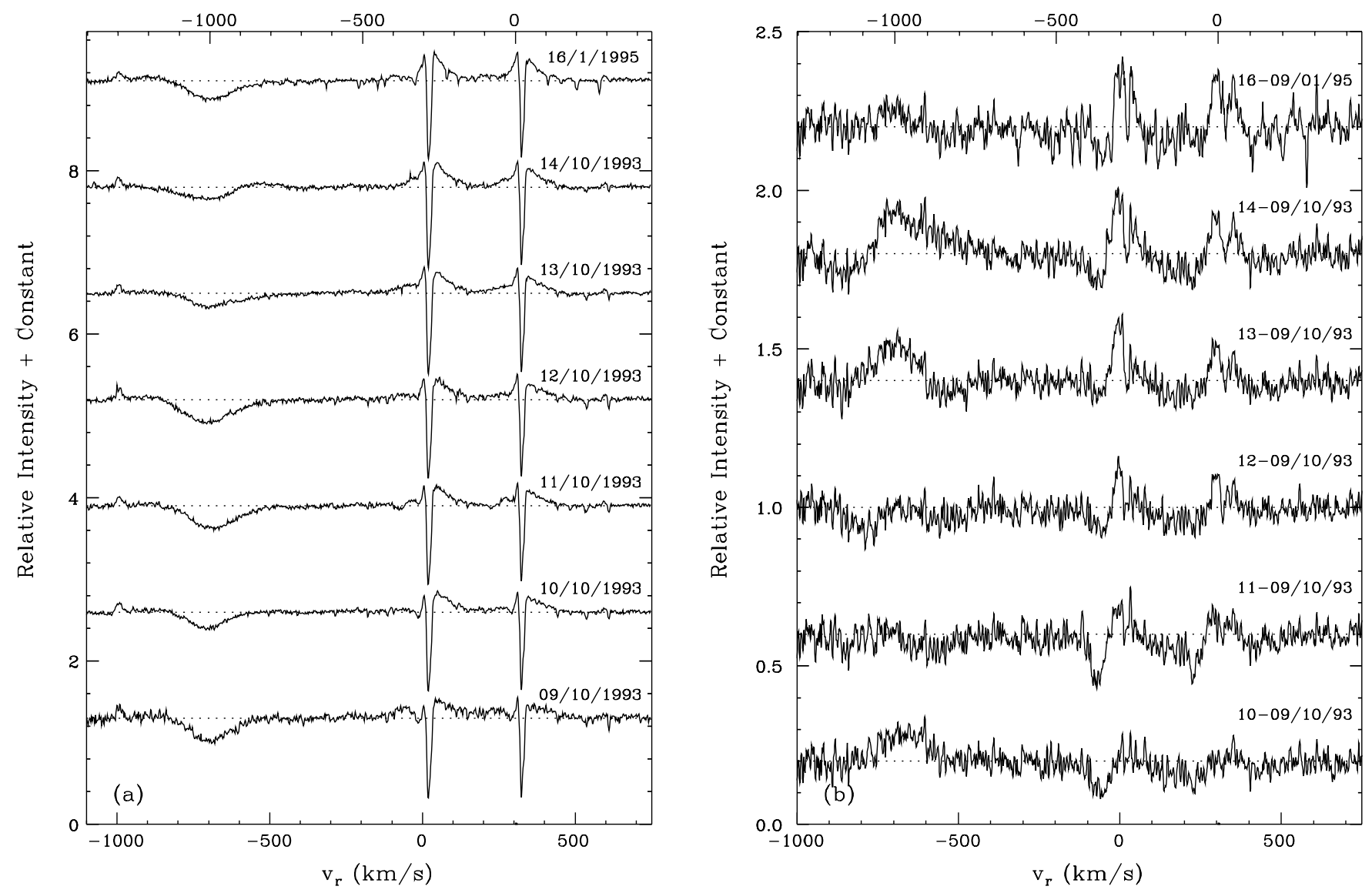

Fig. 9. a) High resolution NaI $D$ spectra of HD 45677, rebinned to heliocentric velocities. b) The same spectra but taken as the difference compared to the one of $09 / 10 / 1993$

indicates gaseous movements and acceleration towards the central star. It would be interesting to monitor HD 45677 daily for a longer time-interval to reconfirm such a mechanism.

In a plot of the differences between the spectra (Fig. $10 \mathrm{~b}$ ), the daily movement is seen as the sharp extra emission on the red side. Furthermore it is clear that an extremely broad and very weak component in the $\mathrm{H} \alpha$ profile changes from a slightly redshifted absorption $(10 / 10 / 1993)$, in fact less emission as it is the difference spectrum with the one taken on $09 / 10 / 1993$, to extra blueshifted emission (11 and 12/10/1993), note the velocity dispersion close to the continuum level in these spectra. Again, this daily change visible as small $V / R$-variations, with an extremely broad velocity dispersion, is either due to the underlying photospheric line or to variations in the wings of the emission profile. Anyway, it seems that infalling gaseous material is followed by extra outflow. This process must be studied further, but it will be related to a region close to the stellar surface.

Another important detection in the $\mathrm{H} \alpha$ profiles are the weaker absorption components and very weak dips, besides the very strong absorption line at $+11 \mathrm{~km} \mathrm{~s}^{-1}$. We detected these components at $-80,-37,+49$ and $+90 \mathrm{~km} \mathrm{~s}^{-1}$. For the weaker ones the error is about $5 \mathrm{~km} \mathrm{~s}^{-1}$. The redshifted components are more prominent.

Table 4. SED fit results for HD 45677 at different $V$ magnitudes

\begin{tabular}{|c|c|c|c|c|c|c|}
\hline $\begin{array}{c}\text { JD } \\
-2440000\end{array}$ & $V$ & $E(B-V)$ & $R_{V}$ & $A_{V}$ & $V_{0}$ & $\begin{array}{c}L_{\star} / d^{2} \\
{\left[10^{-2} L_{\odot} / \mathrm{pc}^{2}\right]}\end{array}$ \\
\hline$\approx 700$ & $8 \mathrm{~m}^{\mathrm{m}} 18$ & $0 \mathrm{~m}^{\mathrm{m}} 27$ & 4.8 & $1 \mathrm{~m}^{\mathrm{m}} 30$ & $6 \mathrm{~m}^{\mathrm{m}} 88$ & 1.16 \\
\hline 2785.51 & $8 \mathrm{~m}^{\mathrm{m}} 49$ & $0 .{ }^{\mathrm{m}} 30$ & 5.6 & $1 .^{\mathrm{m}} 68$ & $6 .^{\mathrm{m}} 81$ & 1.13 \\
\hline 3862.68 & 8. 74 & $0 . \mathrm{m}_{28}$ & $\geq 5.8$ & $\geq 1^{\mathrm{m}} 62$ & $\leq 7^{\mathrm{m}} 12$ & $\geq 0.90$ \\
\hline 4596.77 & 8. 71 & $0 . \mathrm{m}^{\mathrm{m}} 30$ & $\geq 5.8$ & $\overline{\geq} 1^{\mathrm{m}} 74$ & $\leq 6{ }^{\mathrm{m}} 97$ & $\overline{\geq} 0.89$ \\
\hline 4977.45 & 8. 56 & $0 . \mathrm{m}^{\mathrm{m}} 30$ & 5.6 & 1.68 & 6.88 & 1.04 \\
\hline 4977.50 & $8^{\mathrm{m}} 57$ & $0 . \mathrm{m}^{\mathrm{m}} 33$ & 5.4 & $1^{\mathrm{m}} 78$ & $6^{\mathrm{m}} 79$ & 1.17 \\
\hline 4978.46 & $8^{\mathrm{m}} 53$ & $00^{\mathrm{m}} 30$ & 5.6 & $1 .^{\mathrm{m}} 68$ & $6 .^{\mathrm{m}} 85$ & 1.10 \\
\hline 5331.52 & $8^{\mathrm{m}} 55$ & $0 . \mathrm{m}^{\mathrm{m}} 30$ & 5.4 & $1 .{ }^{\mathrm{m}} 62$ & $6 .{ }^{\mathrm{m}} 93$ & 1.03 \\
\hline 5332.42 & $8^{\mathrm{m}} 59$ & $0 . \mathrm{m}_{28}$ & 5.8 & $1 .{ }^{\mathrm{m}} 62$ & 6. 97 & 1.02 \\
\hline 8700.31 & $7^{\mathrm{m}} 96$ & $0 . \mathrm{m}_{31}$ & 4.4 & 1. $\mathrm{m}_{36}$ & $6^{\mathrm{m}} 60$ & 1.23 \\
\hline Grror & $0^{\mathrm{m}} 01$ & $0 \mathrm{~m}^{\mathrm{m}} 02$ & 0.2 & $00^{\mathrm{m}} 05$ & $00^{\mathrm{m}} 06$ & $0 \mathrm{~m}^{\mathrm{m}} 03$ \\
\hline
\end{tabular}



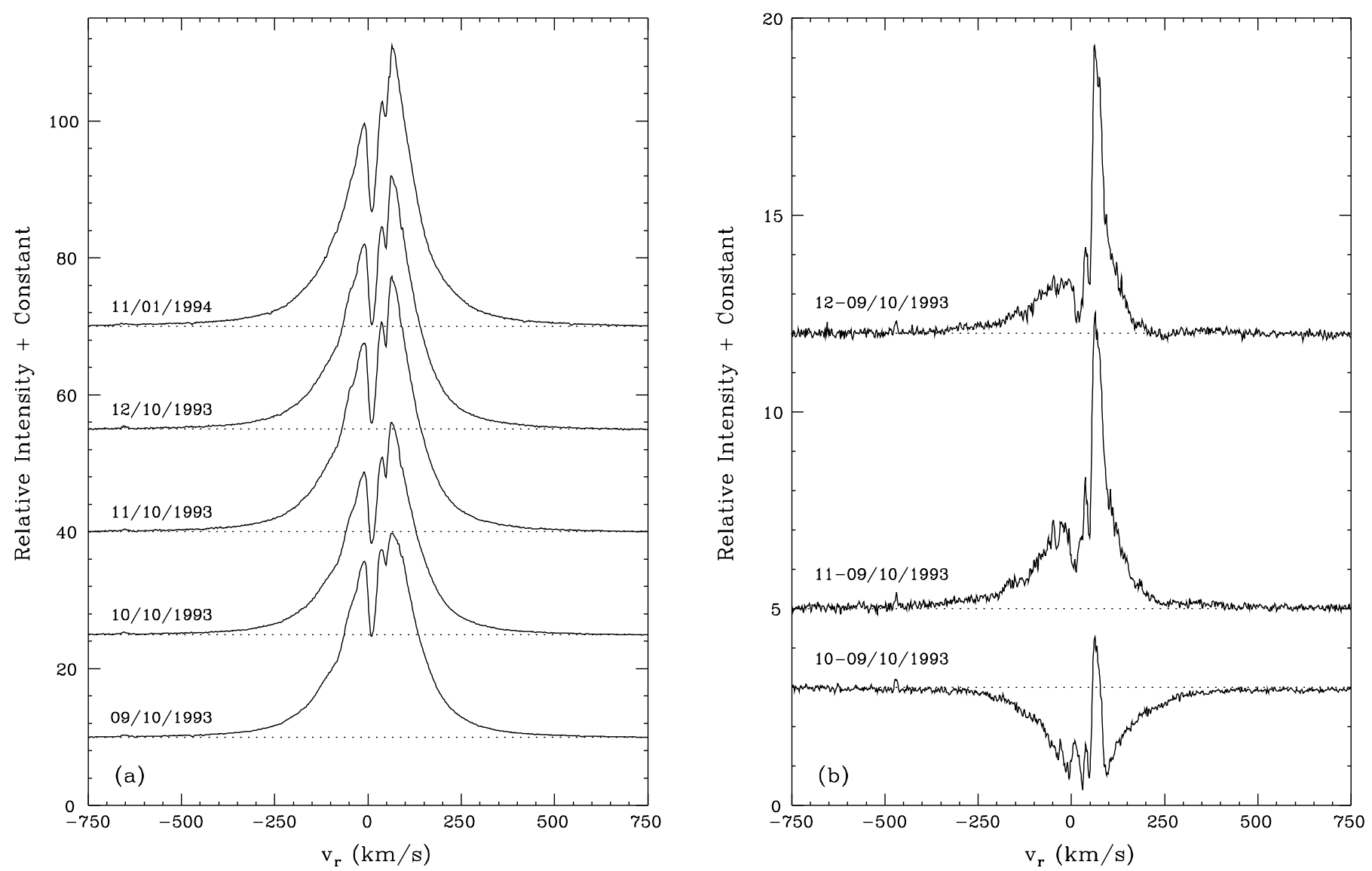

Fig. 10. a) High resolution $\mathrm{H} \alpha$ spectra of $\mathrm{HD} 45677$, rebinned to heliocentric velocities. b) The same spectra but taken as the difference compared to the one of 09/10/1993. Because of saturation effects the spectrum of 11/01/1994 is not shown here

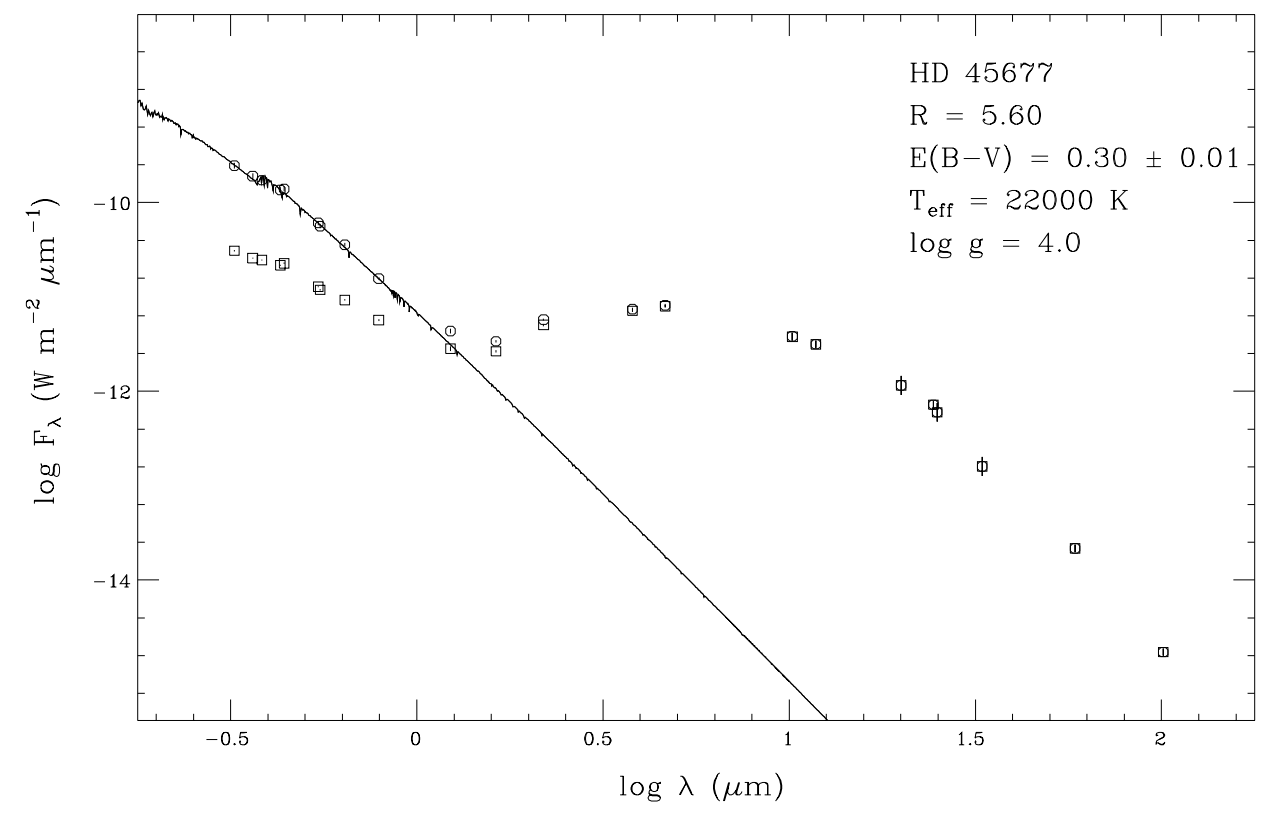

Fig. 11. Typical observed (squares) and extinction-corrected (circles) SEDs for HD 45677. Also shown is the Kurucz model for a B2 V star, fitted to the extinction-free SED 


\section{The spectral energy distributions}

To obtain more information about the variations of the continuum light in the different episodes during the long time scale variations of HD 45677 we have constructed the spectral energy distribution (SED), from the UV to the infrared wavelength ranges, for several $V$ magnitudes. An attempt was made to use as much simultaneous measured data as possible in these SEDs, but simultaneous blue and optical photometry and UV spectrophotometric data were available for a few dates only. Data measured at slightly different times, but at the same stellar brightness were therefore combined into one SED. The SEDs were corrected for interstellar and circumstellar extinction by comparing the observed fluxes to the theoretically observed flux $\left(F^{\text {th }}\right)$ at earth in a photometric system:

$F^{\text {th }}=\int_{0}^{\infty}\left(\frac{\pi R_{\star}^{2}}{d^{2}} F_{\lambda} \operatorname{dex}\left(-\frac{A_{\lambda}}{2.5}\right)\right) S_{\lambda} \mathrm{d} \lambda$.

In this formula $F_{\lambda}$ is the flux emitted by the star per surface unit, $R_{\star}$ is the stellar radius, $d$ is the stellar distance, $A_{\lambda}$ is the total extinction at a given wavelength (in magnitudes) and $S_{\lambda}$ is the transmission function (normalized to unity) of the photometric filter. $F_{\lambda}$ was taken from the Kurucz (1991) model for a B2 V star, assuming solar abundance. $A_{\lambda}$ was obtained from the extinction laws by Steenman \& Thé (1991), using the relation $A_{V}=$ $R_{V} \times E(B-V)$, with $R_{V}$ the ratio of total to selective extinction. A best value of this $R_{V}$, according to the $\chi^{2}$ test, was determined using a trial and error method. Since $R_{\star}$ and $d$ are not accurately known we fitted the parameter $\left(R_{\star} / d\right)^{2}$ to the observed fluxes for each value of $R_{V}$. The results of the fit procedure are listed in Table 4 and a typical resulting SED fit is shown in Fig. 11. In this figure we notice a very good fit to the theoretical Kurucz model in the ultraviolet and in the optical, with a large amount of excess radiation above photospheric levels in the infrared. The presence and appearance of this infrared excess once again indicates the existence of circumstellar dust around HD 45677.

From the SED, we also computed the scaled stellar luminosity using the following formula:

$\frac{L_{\star}}{d^{2}}=4 \pi \int_{0}^{\infty} F_{\lambda, \text { Kurucz }} \mathrm{d} \lambda$,

with $F_{\lambda, \text { Kurucz }}$ the Kurucz model flux, fitted to the extinction-corrected SED. These scaled stellar luminosities are also listed in Table 4.

Again using Eq. (5), we also computed the total luminosity of the infrared excess of HD 45677 by taking $F_{\lambda}$ from a $\beta$-spline fitted to the extinction-corrected SED of HD 45677 minus the corresponding Kurucz model flux. Beyond the last wavelength at which we have observational data, this model was extrapolated with a Planckian curve of $900 \mathrm{~K}$, smoothly joined to the slope of the last spline curve. The resulting scaled luminosity of the infrared excess $\left(L_{\mathrm{IRE}}\right)$ turns out to be about 0.26 $10^{-2} L_{\odot} / \mathrm{pc}^{2}$, or about $28 \%$ of the stellar luminosity. When we also compute the fraction of the stellar luminosity absorbed by dust in the blue and ultraviolet $\left(L_{\mathrm{Abs}}\right)$ by taking the difference between the luminosities of the $\mathrm{Ku}$ rucz model fitted to the extinction-corrected SED and a version of this model reddened with the parameters listed in Table 4, we typically obtain values like $82 \%$ of the stellar luminosity. The ratio $L_{\mathrm{Abs}} / L_{\mathrm{IRE}} \approx 3.0$. The fact that $L_{\mathrm{Abs}} \gg L_{\mathrm{IRE}}$ suggests that either we are seeing a star/disk system at a small inclination angle, or that $\frac{2}{3}$ of the star's total extinction must be interstellar rather than circumstellar as $\frac{1}{3}$ is necessary to produce the re-radiated light as $L_{\mathrm{IRE}}$. In view of the discussion in the previous sections, the first possibility seems more likely.

\section{Discussion of the variations}

\subsection{The photometric variations}

\subsubsection{Long time scale variations/obscuration}

Let us consider the decrease in brightness of HD 45677 from $V=7^{\mathrm{m}} 2$ to $V=8 \cdot \mathrm{m} 8$ as being due to long-term effects (the brighter data-points of the minimum $V=8{ }^{\mathrm{m}} 8$ may be due to shorter time scale variations, which will be discussed in the next two subsections). If we think of this long-term variation as being caused by some obscuration effect, one would need to cover $\sim 77 \%$ of the stellar surface. As was discussed for the pre-main sequence star UX Ori (Bibo \& Thé 1991), these amounts are difficult to realize by intrinsic stellar activities such as star spots, but a slow recovery would explain the relatively slow increase in brightness.

The large changes in $V$ at the long time scale, decennial, and the form of the light curve are also not easily understood by means of obscuration effects like eclipsing by companion(s). The large $\Delta V$ would indicate a nearly total eclipse. Because the complete minimum itself already takes several decennia, the period would be extremely long, indicating a very wide binary or one with a very slow moving eclipsing object. The orbital period of the companion can be much shorter if one explains the long duration of the minimum as one at apastron in a very eccentric orbit, but this can not explain the asymmetric minimum. Another argument against an eclipsing companion is the absence of any periodicity in the radial velocity variations of HD 45677 (Swings \& Allen 1971). However, these amounts of obscuration could be realized by the presence of large $(>1 \mu \mathrm{m})$, presumably circumstellar, dust particles in our line of sight, which will not redden the light of the star, but simply obscure the stellar radiation from the UV to the near infrared.

The colour changes on the long time scale are quite remarkable. The $U-B$ and $B-V$ are getting redder throughout the available data, while the opposite colour changes are seen for $V-R$ and $V-I$ during the 
period of increasing brightness. The complete photometric behaviour can thus not be explained as a simple obscuration effect, as hypothetically discussed above: In that case the getting bluer would be followed by a getting redder again when the star recovers in brightness. In case of pure extra extinction one expects all the colours to get redder. However, if we have a distribution consisting of two distinct grain sizes, as proposed before by SchulteLadbeck et al. (1993), of small grains $(\leq 0.5 \mu \mathrm{m})$ located in the polar lobs and of large grains located in the disk, an increase of both grain populations can produce the observed colour effect. In the polar lobs scattered light will increase and being more reddened by the extra extinction, whereas in the optically thick disk extinction due to larger grains is dominant more efficiently to the longer wavelengths. Such a mechanism effects the visual radiation the less and can explain the colour behaviour. The production of grey grains shortly after a blow-out or evaporation of a cometary body could account for the large obscuration as measured in all pass-bands and the possible getting redder of the $V-R$ and mainly the $V-I$ colours due to extra extinction before the minimum if this was the case.

The difficulty with this scenario is the steady continuation of the $U B V$ colour effects. To account for the required continuous production of small grains out of the larger ones, we need a constant production mechanism to do so, such as a stellar wind, stellar radiation field or mass accretion or a combination of these processes. (a) If accretion is the process, the destruction of the large grains will increase the brightness, small grains will be produced in the polar lobs. Accretion could produce a more compact disk, also explaining the decrease of total infrared flux (Sitko et al. 1994), and therefore getting optically thicker accounting for the extra extinction in the red. In such a model the production of grey grains is then followed by accretion, contributing to the destruction of the large grains mostly close by the central star. (b) Destruction of the grey grains by the stellar wind and the stellar radiation field will create the small grains in outer regions. Scattering at these grains will be added by the scattering in and near a bipolar flow. Radiation and stellar wind pressure could produce a more compressed "ring" of the blown out material to produce the extra extinction. However, when getting thinner reduced extinction due to its expansion must be compensated in this case. The brightening of HD 45677 in this case would be due to evaporation of dust by the stellar radiation and a stellar wind.

Additionally to (a) and (b), if the grey grains were produced by a blow-out around 1950, this material will continue to expand, becoming less efficient in the obscuration. We must note here that the obscuration due to grey grains supports the hypothesis by Sitko et al. (1994). Support for our suggestion that the small grains can also be (continuously) generated by accretion, which is then also seen by an energetic bipolar flow, is given by Grady et al. (1993).
Another model to produce changes in the grain size distribution is to introduce a magnetic field. After the blow-out, the magnetic field recovers in strength, causing a more efficient alignment of the dust particles in time. For this effect one spheroid grain population is needed. Then the colour effects will continue until the original magnetic field is recovered. The brightness variations can be explained in the same way as above. However, this model does not need a bipolar flow of which the existence is still hypothetical.

\subsubsection{Short time scale variations/flickering}

In the paper of Pérez et al. (1993) a strong near-UV excess was reported. If this relatively strong near-UV excess is explained by scattering, as mentioned before in the two grain population model, it will be stable throughout the detected short time scale variations. However, variable extra extinction, due to the production of small grains by accretion, on the scattered and the blue stellar light will effect the near-UV colour more than the blue. If the grains are small enough they will redden the blue colours most efficient, which will result in the colour behaviour as detected. A support for the production of small grains is that, if we explain the photometric short time scale variations as due to variable extinction, their reddening slope indicates $R_{V}$ values $\ll 3.1$.

The shortest time scale variations are then seen as brightening effects in the visual by: re-radiation from the extinction in the blue and near-UV and by the extra accretion luminosity. The resulting "flickering" acts on a time scale of days, as resolved by our new Strömgren 1993 dataset (E) as shown in Fig. 3.

In plots E1-3 of Fig. 3 only a part of one "pulse-like" variation, with a daily trend of diminishing brightness, during the three following nights is seen. Superimposed on this, small brightenings are seen on a time scale of several minutes. As both kinds of "flickering" show the same trends in the colour-magnitude diagrams, see plot E6 of Fig. 3, they are probably of the same origin and are not due to scatter in the data as their errors are of about 0.008 (de Winter et al. 1996) and even less when taken during one night, as is the case. In this case the short time scale "flickering" of minutes are instabilities in the "pulse-like" variations of days. However, here all the colour variations are orientated towards the red at increasing light with a large scatter in the $u-v$ colour, which becomes visible as the points are chronological connected to each other in these plots by lines. We explain these shortest time scale variations as being brightenings due to accretion which will be reflected by grains, being more effective in the less bluer passbands of the observed colours. In this period, 1993, either no extra extinction due to produced small grains seems to be present, which explained the reddening of some colours of the other short time scale variations, as 
before and during the minimum at 1981 or the monitoring period was to short to detect this effect.

The amplitude of the short time scale variations within the different datasets does not seem to vary much, this is best seen in Fig. 1b. The flickering seems therefore present before and after the maximum obscuration around 1981.

We must note that due to inhomogeneities in the circumstellar material there may be moments during which we can look to regions closer to the star which can produce similar effects as mentioned above. In this case variations within a few minutes of 0.015 , in the 1993 Strömgren data, is equivalent with about 1 to $2 \%$ less obscuration of the surface of the central regions. But also in this case these variations are brightening effects.

\subsubsection{Intermediate time scale variations/semi periodic}

Although colour changes of the intermediate time scale variations could only be studied in $B-V$, we can fit them well with the other variations seen when we explain them as obscuration effects by concentrations of dust. The higher extinction then causes the extra reddening with decreasing brightness. If the average grain-size is large this effect will not be significant. A support for this hypothesis is the large $R_{V}$-value of the reddening slope in the $V$ versus $B-V$ diagram of about 7.0-7.5.

Rotation of such dust clouds around the central star could cause the obscurations, extra reddening and recovering in brightness as seen in the lightcurve of HD 45677, Fig. 1b. However, the decrease of brightness in 1989 and 1992 seems to occur slowly compared to the getting brighter in 1991. This can be explained as due to the superpositions of the brightening in the long time scale variations. Another explanation for the intermediate time scale variations is the production of large grains at certain moments. The getting brighter is then due to the subsequent destruction of such grains or the rotating away of these regions from our line of sight.

As we have seen that the short time scale variations are brightening effects, the same could be true for the intermediate time scale variations. In that case we have maxima during 1988 and 1992. Just before these maxima we see in Fig. 1b that the "flickering" amplitude is relatively high, followed by a decrease during the maxima and after that getting larger again. In this picture there seems to be a connection between the short time scale accretion and the intermediate time scale variations. The latter can be explained by infall of circumstellar material which lasts 1-2 years and which produces minima. Then the dense obscuring material will be destroyed by accretion as seen by the short time scale variations. The infall explanation will not violate the $B-V$ colour effects for the intermediate time scale variations, it is still due to extra CS extinction. Here the brightening and fading are simply due to the accretion efficiency and do not need a complex explanation.
Continued monitoring, both in magnitude and in colours, of HD 45677 is necessary to select the correct mechanism.

\subsubsection{Variations in $R_{V}$ values}

From Table 4 we see that the obtained $R_{V}$ values range from 4.4 to $\geq 5.8$, and vary roughly with $V$, resulting in a nearly constant intrinsic stellar brightness. Also note that the maximum value of $R_{V}$ in the grid of extinction curves by Steenman \& Thé (1991) is 5.8 and our SED fit resulted in that value for several fits near the state of minimum brightness of HD 45677 around 1980. Therefore, it seems likely that the true value of $R_{V}$ near minimum brightness will be somewhat higher than 5.8 .

These variations of the $R_{V}$ values with stellar brightness had previously also been found by Brown et al. (1995) from UV extinction curves, in which they found the value of $R_{V}$ for the total extinction towards HD 45677 to vary between 4 and 5. Brown et al. (1995) did also note that the value of $R_{V}$ for individual dust clouds around HD 45677 must be much larger $\left(R_{V}>7\right)$, see also Sect. 6.3.

From the variations of $R_{V}$ with brightness we conclude also that the long time scale variations are not caused by intrinsic variations of the central star, but to an external effect. A change in the average particle size in the circumstellar dust would indeed change the circumstellar extinction law. Note that the derived $R_{V}$ values describe a theoretical extinction law of Steenman \& Thé (1991) which is taken to be the observed average extinction law from the UV to the near-IR, but wavelength-dependent deviations of this law due to other grain populations can be expected.

\subsection{The spectroscopic behaviour}

\subsubsection{Long time scale variations}

Concerning the long time scale variations, the major point is that the observations and conclusions of Swings et al. (1980) and all the observations made since 1928 (Swings \& Allen 1971) are confirmed. The lines as detected in the new spectra are in majority very constant in relative strength, radial velocity and profiles, when detectable, compared to those seen before.

An exception is the FeII spectrum. As reported the Fe II lines are double peaked and more dispersed that the [Fe II] lines. The Fe II lines are thus formed in another region, closer to the star with gases moving at higher velocities and therefore with wider emission profiles. Since there is no central re-absorption, the outer material is optically thin for this radiation. This leaves the double peaked Fe II to be explained by a rotating disk or ring with $16 \mathrm{~km} \mathrm{~s}^{-1}$. In all observations made Fe II shows an emission spectrum including the forbidden transitions. Note the remarkably stable average radial velocity of $18 \pm 7 \mathrm{~km} \mathrm{~s}^{-1}$ (Swings \& Allen 1971). The so-called stellar lines are stable at a 
somewhat higher displacement, $24 \pm 7 \mathrm{~km} \mathrm{~s}^{-1}$. This is explained by outflowing material as the bright lines show absorption cores in their violet wing and low $V / R$ values for most hydrogen lines (Merrill 1928).

In the 1992 intermediate resolution spectra the double peaked profiles show separations of about $40-60 \mathrm{~km} \mathrm{~s}^{-1}$, which is somewhat higher than in the spectra taken before the photometric minimum. This can be explained by assuming that the Fe II lines are formed in the disk region which contains gas, moving faster than before 1982, and dense enough for some self-absorption, or with a higher rotation velocity. The stable identified nebular lines must be produced in a region further out, but in a region which is not much flattened and of which the velocity dispersion is low as they are not as broad as the Fe II lines.

In combination with the dramatic photometric changes (thus in the continuum), we conclude that these do not seem to affect the spectral lines much. The mechanism causing the photometric changes is thus either separated from the line forming regions or not influencing the amount and other properties of the gaseous material. Furthermore, indications for the existence of a disk like region by spectral lines and their profiles are based on data taken and models proposed far before the 1950 event.

\subsubsection{Short time scale variations}

Although the long time scale variations are only seen for the photometric behaviour, short time scale changes in line profiles were reported above as well as before in many papers (Merrill 1928; Swings et al. 1980). This indicates that HD 45677 was already active in the beginning of this century. To have a better view of such behaviour today we monitored HD 45677 for several days around the $\mathrm{NaI} D$ lines, including $\mathrm{He} \mathrm{I}$, and $\mathrm{H} \alpha$.

First of all we detected the Na I $D$ lines to be in emission. If we take a one atom thick shell of neutral sodium at a distance of roughly 1 AU from a B2 V central star, all of this material will be evaporated and ionized within 1 second. The presence of $D_{1}$ and $D_{2}$ emission profiles, therefore, indicates the existence of cool material far away from the hot central star. On the one hand the slight $\mathrm{P}$ Cygni nature of these lines proves the existence of outflowing radiating cool gas from the central star. On the other hand, the strong absorption cores at $20 \pm 2 \mathrm{~km} \mathrm{~s}^{-1}$ indicate that a significant part of this material is at zero velocity, adopting a systemic velocity of $20 \mathrm{~km} \mathrm{~s}^{-1}$. The equal strength of the $D_{1}$ and $D_{2}$ absorption components, which are stable throughout all spectra, indicates the saturation of these components and therefore the presence of a high column density of the circumstellar material in the disk, of which the existence was indicated before.

Dense outflowing material, released in the disk, could be present as indicated by the blueshifted absorption lines being dynamical on a daily time scale. The outer sides of the disk can then be responsible for the wide sodium emission. It is not clear what the central velocity of this broad emission line is and therefore it remains unclear if the strong absorption at systemic velocity could be due to self-absorption in the outer part of the disk. Furthermore, collisions of the outflowing material with the accreting or study disk material at rest, causing inhomogeneous dense structures, could also be the origin of the blueshifted narrow absorption components; see also Israelian et al. (1996). This could explain the wide variety of the velocities, strength and duration of visibility of such components. However, the enrichment of the disk with new material could also act as the environment for the formation of the necessary large grains although the destruction of the material could be more significant, as discussed before.

It must also be noted that the variable brightness of HD 45677 could be the cause of the slight differences in the Na I $D$ emission strength, when produced in the outer parts of the circumstellar disk.

The variation in the strength of the He I absorption line can be partly caused by obscurations of the central star as the photometry indicates such time scale variations. However, the variations are also seen in the line profiles and are too strong to be accounted for by the relatively weak photometric changes on the daily variations. As compared to 09/10/1993, Fig. 9b, we see especially on 13 and 14/10/1993 extra "emission". Note that this component is asymmetric, the red side is extended to higher velocities, especially on $14 / 10 / 1993$, when this relative emission is at its strongest. The extra emission or less absorption at the red side, exactly the opposite as what we see for the Na I $D$ lines in the same spectra, must originate from infalling material very close to the stellar surface. This could also produce collisionally ionized gas when it coincides with the inner disk material. Because of the redshifted origin, this component of a hot wind, can not be accounted for by a bipolar outflow. The existence of this emission component and the short time scale indicates a strong dynamical process close to the central star. This infall process is necessary for the existence of a bipolar flow anyway.

Because of the co-variations of the emission components that are red- and blueshifted for the He I and $\mathrm{Na}$ I $D$ lines, respectively, there must be some common origin. Although the processes are of different velocity sign and the line formation region must be different we assume that on moments that dust-concentrations obscure the central regions and the star, we will not see much of the He I emission region but only a contribution of the stellar spectrum. However, also parts of the disk itself will be obscured and these high dust densities can also cause the narrow absorption lines in $\mathrm{Na}$ I $D$. It would be interesting to test this hypothesis by simultaneous high resolution spectroscopy and photometry.

In addition to these effects Israelian et al. (1996) reported emission at the violet side of the He I profile as well at times when its central absorption is at its strongest. 


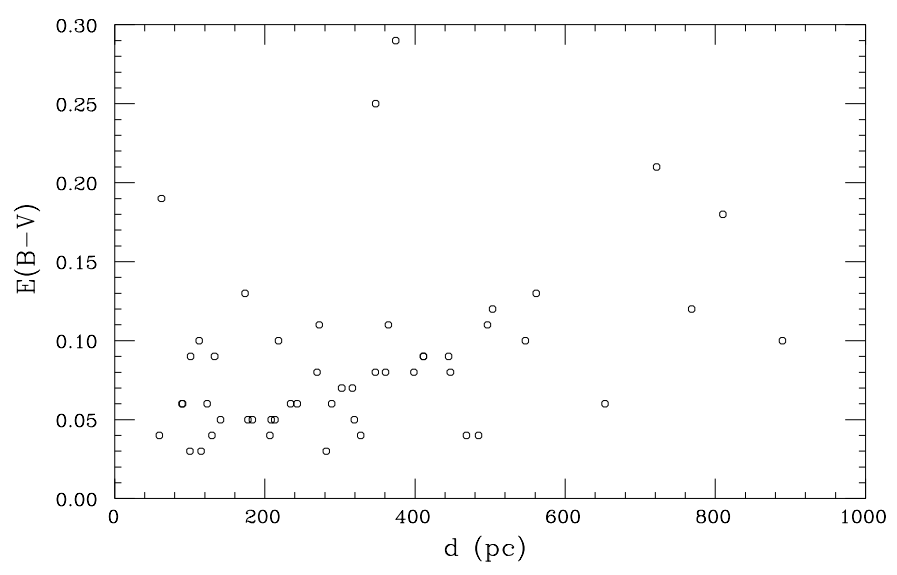

Fig. 12. $E(B-V)$ versus photometric distance for stars from the Hipparcos Input Catalogue within $1^{\circ}$ of HD 45677

\section{Evolutionary status}

The group of $\mathrm{B}[\mathrm{e}]$ stars, to which HD 45677 belongs, is not very homogeneous. It contains young stellar objects, such as Herbig Be stars, but also evolved objects, such as postAGB stars, Luminous Blue Variables (LBVs) and planetary nebulae (Thé et al. 1994). Some of these stellar groups even occupy overlapping regions in the HR-diagram, which makes it even more difficult to classify such objects. To obtain a star's position in the HR-diagram we must know its distance, which is very uncertain for HD 45677. Sitko et al. (1994) argued that the UV extinction curve of HD 45677 in 1992 is compatible with interstellar extinction over a distance of $900 \mathrm{pc}$. At this distance HD 45677 would be at approximately the same distance as the outer ridge of a large molecular complex that stretches from the Mon R2 cloud to the CMa OB1 complex (Maddalena et al. 1986).

However, since our line of sight towards parts of Canis Majoris is known to be almost devoid of interstellar extinction (e.g. Bruhweiler 1994), the use of an average distanceinterstellar extinction relation by Sitko et al. (1994) may yield erroneous results. Therefore, we constructed a graph of $E(B-V)$ versus photometric stellar distance for stars from the Hipparcos Input Catalogue, located within an $1^{\circ}$ circle around HD 45677. This plot is shown in Fig. 12. From this plot we derive a maximum distance of $\approx 1.5 \mathrm{kpc}$ towards $\mathrm{HD} 45677$, by assuming that its $E(B-V)$ of 0.30 is completely due to interstellar extinction.

Furthermore, we can compute a photometric distance towards HD 45677 by assuming that it is located on the zero-age main-sequence (ZAMS) and by assuming that there is no circumstellar obscuration present at the maximum recorded brightness of HD 45677 by Swings \& Swings (1972). If we also assume that its $B-V$ at that time was not very different from the minimum of the range shown in Fig. 2, and use the appropriate parameters for a B2
V star from Schmidt-Kaler (1982), the resulting ZAMS distance is $550 \mathrm{pc}$. Under the same assumptions we computed the maximum intrinsic luminosity of HD 45677 by adopting the maximum distance of $1.5 \mathrm{kpc}$, resulting in $L_{\star, \max }=3.710^{4} L_{\odot}$.

A HR-diagram with the position of $\mathrm{HD} 45677$, computed using the ZAMS distance of $550 \mathrm{pc}$, the distance of 900 pc by Sitko et al. (1994), and the maximum distance of $1.5 \mathrm{kpc}$ is shown in Fig. 13. In Fig. 13a the pre main-sequence evolutionary tracks and the birthline by Palla \& Stahler (1993) for a proto-stellar accretion rate of $10^{-5} M_{\odot} \mathrm{yr}^{-1}$ are also plotted, whereas in Fig. 13b we plotted the post main-sequence evolutionary tracks by Maeder \& Meynet (1988). As can be seen from Fig. 13a, of the three positions of HD 45677 in the HR diagram, the one computed using the ZAMS distance of $550 \mathrm{pc}$ is the only one which falls below the birthline. This implies that if HD 45677 is a young object, as suggested by Grady et al. (1993), its distance will be close to its ZAMS distance of $550 \mathrm{pc}$. In that case the star cannot be formed in the molecular cloud suggested by Sitko et al. (1994), leaving us with the question where the star formation region is. However, as shown by Palla \& Stahler (1993) a higher proto-stellar accretion rate will result in a birthline which will intersect the ZAMS at considerably higher masses. But for masses like for HD 45677 still not more than $10^{5} \mathrm{yr}$ is needed to reach the ZAMS.

If we assume that HD 45677 is in fact an evolved object, we do not have these problems. In that case the star might very well have formed in the molecular complex suggested by Sitko et al. (1994), but a few times $10^{7}$ years ago. Let us first consider several options of evolved objects.

Eruptive behaviour is known for massive stars close to the Humphrey-Davidson (HD) limit, such as LBVs (Humphreys et al. 1989). In the case of HD 45677 the temperature did not change as much as seen for LBVs in such a situation, and would be too high to fit a position close to the HD limit within a reasonable distance. Note here that indications of the luminosity class of HD 45677 are III or IV (Pérez et al. 1993). However, the existence of the AG Car ring nebula is also not well explained by instabilities close to the HD limit as its luminosity class is still not exactly known (Humphreys et al. 1989). Other evolved objects that undergo phases of mass ejections leading to the formation of dusty disks as visible in the near- and far-IR are post-AGB stars and Symbiotics. HD 45677 is too hot to be a post-AGB star, but it could be in a certain transition phase of PNs. The presence of a companion could be the origin of the perturbations as detected. At the moment there are not even traces of such a companion.

Forbidden lines of [O I], [N II], [S II] and [Fe II] indicate the existence of a low density region. The electron density of this region, $N_{\mathrm{e}} \approx 810^{3} \mathrm{~cm}^{-3}$, is determined by the ratio of the $[\mathrm{S} \mathrm{II}]$ lines and is high compared to most nebulae (Osterbrock 1974). Because HD 45677 is too "cool" to ionize gas that emits lines of [OIII], we derived the 
electron temperature by the $[\mathrm{N}$ II $]$ lines and determine $T_{\mathrm{e}} \sim 610^{4} \mathrm{~K}$, which is very high. The $N_{\mathrm{e}} / T_{\mathrm{e}}^{1 / 2}$ value of 30 is not far from those of some planetary nebulae (Osterbrock 1974). Since the temperature of HD 45677 is relatively low, these values should indicate that an extended region should be visible. However, on our images HD 45677 appears unresolved, so it seems unlikely that HD 45677 is such a planetary nebula. The values also agree with the conclusion of Swings (1973) who mentioned this region as being of considerable density, but with a low temperature based on the absence of some forbidden lines like [S II]. Swings (1973) reported the occurrence of [N II] lines, now the $[\mathrm{S} \mathrm{II}]$ are also detected, clearly indicating the ongoing evolution of this region.

However, one of the most peculiar lines is the very strong and narrow blueshifted $\mathrm{H} \alpha$ absorption component at $9 \mathrm{~km} \mathrm{~s}^{-1}$ relative to the systemic velocity. If we propose that this concentration of considerable density of gas is distributed in a ring-like structure, indicated by its low velocity-dispersion, it might originate from the 1950 event. At a minimum distance of $500 \mathrm{pc}$, the angular size of this ring will be about $0 . \prime 2$ and indeed not yet visible.

As the evolved status of HD 45677 is also not clear we return to the hypothesis that HD 45677 is still in a premain sequence phase. This option has some support by the new $v \sin i$ value of $70 \mathrm{~km} \mathrm{~s}^{-1}$ (Israelian et al. 1996), a value similar to several comparable PMS objects, and the detection of blueshifted He I emission as is reported for several HAeBe stars by Böhm \& Catala (1995). These findings are probably not unique for HAeBes. Furthermore, the evaporation of cometary-like bodies in the vicinity of some Herbig Ae type stars, the UXOR-group, has recently been discovered (Grinin et al. 1994 and 1996, de Winter et al. 1995 and de Winter 1996) and show some typical behaviour in colour and variation in line profiles. A colour behaviour due to pure extinction, in the minima, or the so-called "blueing effect", during the deep minima, as due to revolving dust clouds in a proto-planetary disk, are not seen for HD 45677. Also the UXOR-group shows clear evidence for infalling evaporating comet-like bodies by redshifted absorption components in the NaI $D$ lines with co-variations in the $\mathrm{He}$ I and $\mathrm{H} \alpha$ profiles on a daily time scale. The suggested evaporation of a large cometarylike body around 1950 should have produced similar line profiles. However, no dramatic changes in the mentioned lines have been noticed, although the resolution could be a limiting factor. Also, the time scale and amplitude of the minimum is comparable to those for objects in the UXOR-group.

Finally, when HD 45677 is indeed a product of a molecular complex related to the Mon $\mathrm{R} 2$ cloud and the CMa OB1 complex (Maddalena et al. 1986), it would be interesting to know whether it is still young enough for postnatal circumstellar material to be the origin for the dynamical properties observed. HD 45677 would then be an isolated young object being only some $10^{5}$ yrs old, similar to isolated HAe objects, such as HD 104237, HR 5999 and HD 163296. But, in these cases evidences of their relative youth, up to several times $10^{7} \mathrm{yrs}$, are still seen in their near surroundings such as $\mathrm{T}$ Tauri stars.

To give the final answer to the question of the evolutionary statues of HD 45677, a more accurate distance determination than the one presented here is necessary. If all goes well, this will become available soon, since this object is one of the primary targets for the Hipparcos astrometric satellite.

\section{Concluding remarks}

From an intensive study of the photometric and spectroscopic properties of HD 45677, including published data as well as own observations, we have detected many different variations and properties. We have tried to explain these variations, from short to long time scales. Different mechanisms must produce the variations, each with their own characteristics. Furthermore, we have discussed different options for the evolutionary status of HD 45677. Although the properties of HD 45677 are disputable we can make the following concluding remarks:

- Photometric variations and spectroscopic properties prove the hypothesis of a significant disruption of material around HD 45677. By extrapolation to the maximum brightness as measured by Swings \& Swings (1972), before any large photometric changes occured, we determined this event took place around 1950. The minimum brightness at $V=8.8$ was reached around 1981. Linear extrapolation of the current increase of brightness indicates that the maximum brightness of $V=7^{\mathrm{m}} 2$ will be reached again about the year 2020 .

- During this long time scale obscuring effect we observed that the colours do not change significantly at both the obscuration and brightening phases. To explain this, formation of large grey grains is proposed which caused the strong obscuration of HD 45677 just after the 1950 event. The existence of such grains was also detected by extinction studies of Sitko et al. (1994) and already proposed by Low et al. (1970). We suggest that the origin of this material around 1950 is either due to the evaporation of a large cometary-like body, equal to less dramatic but similar as events for UX Ori (Grinin et al. 1994), or caused by a blow-out of material. Probably this blow-out was not extremely violent because of a fall back of material causing accretion effects.

- The relatively small and continuous changes of the colours during the period 1971-1993 are remarkable because of the opposite sign of the blue colours versus the red colours. If part of the erupted material falls (back) in the direction of the central star, a more compact inner disk region will cause higher extinction for the red colours, while the blue colours 

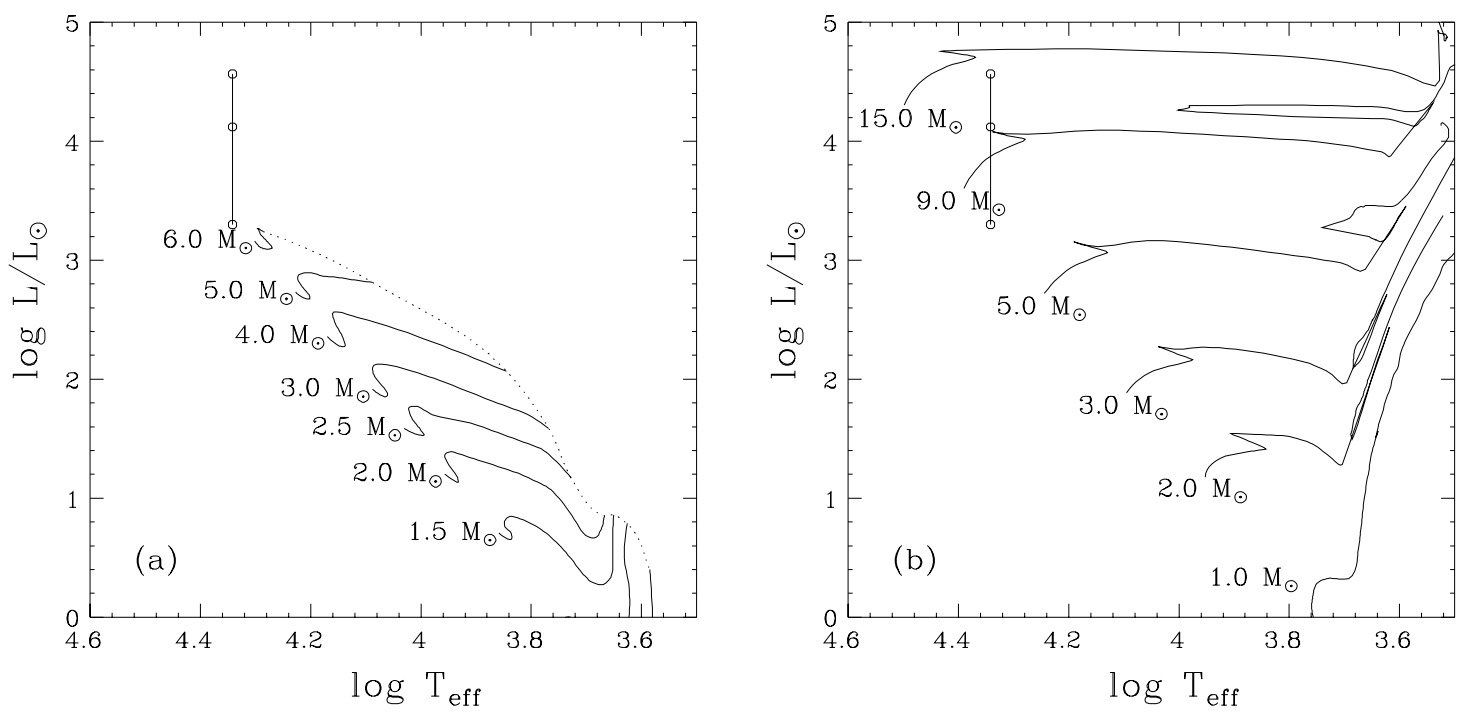

Fig. 13. HR-diagram with the position of HD 45677 assuming a distance of $550 \mathrm{pc}$ (bottom), 900 pc (center), and $1.5 \mathrm{kpc}$ (top). a) With the pre main-sequence evolutionary tracks and birthline by Palla \& Stahler (1993), b) With the post main-sequence evolutionary tracks by Maeder \& Meynet (1988)

can be compensated with the production of small grains by accretion probably being visible in the bipolar flow.

- Variations on a time scale of days down to several minutes are detected. These short time scale variations are explained by brightenings or "flickerings" in the brightness as well as in the colour due to accretion. To understand the colour variations a production of small grains by accretion is necessary. The existence of small grain particles, producing scattering, is detected by an UV-excess (Pérez et al. 1993) which could be due to a bipolar flow (Schulte-Ladbeck et al. 1993), maybe produced but certainly enriched by the accretion itself. This accretion supports the explanations for the origin of the long time scale variations in which the presence of accretion is suggested.

- Photometric variations on an intermediate time scale are also detected and well known. These variations need more observations to be well explained. They seem to exist before and after the minimum in 1981 but their changes of 0.4 within a few years were probably absent before 1930. The intermediate time scale variations could be produced by revolving "dust clouds" or by shock fronts. In the latter case the blow-out hypothesis suggests certain eruptions. The occurrence of larger variations and the slightly getting brighter just before the strong fading around 1950 would support this. Then the intermediate time scale variations following the 1981 minimum are more probably due to infall. Support for this is that the brightening seems to occur in certain "waves" and dust destruction by accretion is seen by the higher amplitudes of the short time scale variations. The latter can vary then up to about $0.1 L_{\star}$ within a few days. Obscurations by "dust clouds" will also cause completely different colour effects as well discussed for the HAe UXOR type objects (e.g. Grinin et al. 1994 and references therein).

- The fact that $R_{V}$ changes from 4.8 in 1970 to $\geq 5.8$ in 1981 and back to 4.4 in 1992 means that the average particle size in the circumstellar matter responsible for the extinction increased from 1970 to 1982 and decreased from 1982 until 1992.

Probably the large grains were directly formed after 1950, due to a blow out or by the evaporation of a cometary-like body. Then the combination of the ongoing wind/outflow, coinciding with the previously erupted material and the pressure at the edges of the shockfront can produce larger grains by collision. The increase of the average grain size seems then to be most effective in obscuring the central star.

At a certain point, $\sim 1982$, the large grains were destroyed again. Infall of material seems to occur in waves, the intermediate time scale variations, which stimulates the accretion as seen as the higher amplitudes of the short time scale variations. As a result, the average grain size will decrease, producing the observed lower $R_{V}$ values and giving rise to brightening of the object again, apart from the effect of an expanding ring of material. A dramatic colour change on the longer time scale due to the production of these smaller grains is not necessary when the material is either effectively evaporated or destroyed and blown away in a bipolar flow. It supports the conclusion of Sitko et 
al. (1994) of a decrease of the total mass of the star's circumstellar dust envelope.

- Spectroscopically we have noticed that forbidden lines are narrow compared to other observed emission lines and are well centered on $20 \mathrm{~km} \mathrm{~s}^{-1}$. This velocity is also valid for the narrow absorption components of Na I $D$ and CaII $K$ lines and the central velocity of the Fe II spectral lines. We adopted therefore a systemic velocity of $20 \mathrm{~km} \mathrm{~s}^{-1}$. Relative to this velocity in- and out-flow of gaseous hot and cool material are seen.

- Remarkable blueshifted absorption lines of Na I $D$ are detected at -10 to $-20 \mathrm{~km} \mathrm{~s}^{-1}$. They are shifted with about 30 to $40 \mathrm{~km} \mathrm{~s}^{-1}$ compared to the systemic velocity. They are probably caused by material swept up by the radiation pressure and stellar wind. This cool grain material will collide with the outer disk causing extra cogglumeration and obscurations on short time scales. Although the intermediate time scale variations can be explained in a similar way, by material moving away from the central star, a more plausible explanation is accretion activity. During the observations of the high resolution time series, this activity was decreasing, see Fig. 10b.

- H $\alpha$ profiles are detected to contain absorption components on the large emission profile at certain velocity regimes of which the red components are the most prominent. The $\mathrm{H} \alpha$ profiles are therefore interesting as they show velocity regimes of both infalling and outflowing material. Although the infalling gaseous material seems to be of higher densities, the strongest narrow absorption core is blueshifted with $9 \mathrm{~km} \mathrm{~s}^{-1}$ with respect to the systemic velocity adopted as $20 \mathrm{~km} \mathrm{~s}^{-1}$. We might expect that especially this very strong $\mathrm{H} \alpha$ component originates from a very dense gaseous ring of which the velocity-dispersion will be as low as is observed. It could be questioned if this "wave" might exist for about 40 years.

- Accretion close to the stellar surface is indicated by the $\mathrm{He}$ I and $\mathrm{H} \alpha$ short time scale variations, probably followed by a reaction of outflowing gas. This dynamical process could replenish the bipolar flow. It could be questioned if the spectroscopic short time scale variations show co-variations to the "flickering" as being of a similar, daily, time scale. Furthermore, the line variations are very different from those seen for many of the UXOR type stars (Grinin et al. 1994 and 1995) for which redshifted absorption components of the $\mathrm{Na}$ I $D$ are detected as evidence of the evaporation of cometary-like bodies.

Observations as for the He I lines are also reported by Israelian et al. (1996), whose conclusions do support our detection of accreting gas.

- Outflow of cool material is seen by emission lines that are stronger towards the blue as in $\mathrm{Mg}$ I and in $\mathrm{NaI} D$. The strong absorption cores of the Na I $D$ and Ca II $K$ indicate the presence of an optically thick disk at zero-velocity. This suggests that this disk was already present and is now enriched by the 1950 event.

- The presence of a disk before 1950 is also indicated by other emission lines which are stable throughout the large and long-term photometric variations and even far before 1950. In this case the 1950 event is due to an instability which is probably not the first one. Evidence of "ancient" activities, probably close to the stellar surface, were seen from 1926 on (Swings 1973) until now by activity in several of the Balmer lines.

- The evolutionary status of HD 45677 is discussed extensively. However, no strong conclusions can be drawn about its age. HD 45677 seems to fit best in the picture of Zickgraf \& Schulte-Ladbeck (1989) of non-luminous $\mathrm{B}[\mathrm{e}]$ stars in contradiction to the $\mathrm{B}[\mathrm{e}]$ supergiants of the Magellanic clouds (Zickgraf et al. 1986). Probably in the case of HD 45677 the dust does not occur by a dense, slow expanding equatorial wind, but by eruptions. HD 45677 could therefore act as a key object to understand the reason of dust formation rather than to evolve to an early-type emission line star without dust.

Acknowledgements. The authors wish to thank Dr. B. Gilbert for providing the photometric data of HD 45677 in the Geneva system and R. van Duuren for his assistance to obtain the CAT/CES observations. We are thankful to Dr. J.P. Swings for his help, inspiration and patience in tracing some unpublished observations and to Dr. G. Israelian, Dr. C.A. Grady, Dr. M.R. Pérez and the referee for their many useful comments. This research has made use of the Simbad data base, operated at CDS, Strasbourg, France.

\section{References}

Allen D.A., 1973, MNRAS 161, 145

Allen D.A., Swings J.P., 1976, A\&A 47, 293

Bergner Y.K., Miroshnichenko A.S., Yudin R.V., et al., 1995, A\&AS 112,221

Bessell M.S., 1979, PASP 91, 589

Bibo E.A., Thé P.S., 1991, A\&AS 89, 319

Böhm T., Catala C., 1995, A\&A 301, 155

Bruhweiler F.C., 1994, in "Frontiers of Space and GroundBased Astronomy: The Astrophysics of the 21st Century", Longair M.S., Kondo Y., Wamstekker W. (eds.). Kluwer, Dordrecht, The Netherlands, p. 289

Burnichon M.L., Chalonge D., Divan L., Swings J.P., 1967, J. Obs. 50, 391

Cameron R.C., 1966, Georgetown Obs. Monograph 21

Ciatti F., D’Orico S., Mammano A., 1974, A\&A 34, 181

Coyne G.V., Vrba F.J., 1976, ApJ 207, 790

Dickinson D.F., Snyder L.E., Brown L.W., Buhl J., 1978, AJ 83,36

Doazan V., Sedmak G., Barylak M., Rusconi L., 1991, "A Be star atlas of far UV and optical high-resolution spectra", ESA-SP 1147

Fajardo-Acosta S.B., Knacke R.F., 1995, A\&A 295, 767

Feinstein A., Garnier R., Vogt N., et al., 1976, A\&A 51, 269

Gilbert B., 1994 (personal communication) 
Grady C.A., Bjorkman K.J., Shepherd D., et al., 1993, ApJ 415, L39

Grady C.A., Pérez M.R., Thé P.S., 1994, ASP Conf. Ser. 62, 409

Grinin V.P., Thé P.S., de Winter D., et al., 1994, A\&A 292, 165

Grinin V.P., Kozlova O.V., Thé P.S., Rostopchina A.N., 1995, A\&A 309, 474

Halbedel E.M., 1989, PASP 101, 999

Halbedel E.M., 1991, IAU Inform. Bull. Var. Stars 3602, 1

Haupt H.F., Schroll A., 1974, A\&AS 15, 311

Horne K., 1986, PASP 98, 609

Humphreys R.M., Lamers H.J.G.L.M., Hoekzema N., Cassatella A., 1989, A\&A 218, L17

Israelian G., Friedjung M., Graham J., et al., 1996, A\&A (in press)

Jaschek M., Jaschek C., Andrillat Y., 1993, A\&AS 97, 781

Jaschek M., Jaschek C., Andrillat Y., Houziaux L., 1992, MNRAS 254, 413

Kilkenny D., Whittet D.C.B., Davies J.K., et al., 1985, South African Astron. Obs. Circ. 9, 55

Kurucz R.L., 1991, Precision photometry: Astrophysics of the galaxy. In: Davis Philip A.G., Upgren A.R., Janes K.A. (eds.). L. Davis press, Schenectady, New York

Le Squeren A.M., Sivagnanam P., Dennefeld M., David P., 1992, A\&A 254, 133

Low F.J., Johnson H.L., Kleinmann D.E., Latham A.S., Geisel S.L., 1970, ApJ 160, 531

Maddalena R.J., Morris M., Moscowitz J., Thaddeus P., 1986, ApJ 303, 375

Maeder A., Meynet G., 1988, A\&AS 76, 411

McGregor P.J., Hyland A.R., Hillier D.J., 1988, ApJ 324, 1071

Mendoza E.E., 1958, ApJ 128, 207

Merrill P.W., 1928, ApJ 67, 405

Merrill P.W., 1952, ApJ 116, 501

Merrill P.W., Burwell C.G., 1933, ApJ 78, 87

Nyman L.A., Booth R.S., Carlstrom U., et al., 1992, A\&AS 93, 121

Osterbrock D.E., 1974, Astrophysics of Gaseous Nebulae, Freeman and Company, San Francisco

Oudmaijer R.D., Drew J., 1995, in Proc. ESO Workshop on: "The Role of Dust in the Formation of Stars", Siebenmorgen R. and Käufl H.U. (eds.) (in press)

Palla F., Stahler S.W., 1993, ApJ 418, 414

Penston M.J., 1973, MNRAS 164, 133

Pérez M.R., Grady C.A., van den Ancker M.E., et al., 1993, in "Frontiers of Space and Ground-Based Astronomy, The Astrophysics of the 21st Century", Longair M.S., Kondo Y., Wamstekker W. (eds.). Kluwer, Dordrecht, The Netherlands, p. 563

Pérez M.R., 1994 (personal communication)

Rufener F., Nicolet B., 1988, A\&A 206, 357

Schmidt-Kaler Th., 1982, Landölt-Bornstein Catalogue VI/2b

Schulte-Ladbeck R.E., Shepherd D.S., Nordsieck K.H., et al., 1993, ApJ 401, L105

Simpson J.P., 1991, ApJ 368, 570

Sitko M.L., Halbedel E.M., Lawrence G.F., Allyn Smith J., Yanow K., 1994, ApJ 432, 753

Sorrell W.H., 1989, MNRAS 241, 89

Steenman H., Thé P.S., 1991, Ap\&SS 184, 9

Swings J.P., 1971, A\&A 26, 443

Swings J.P., 1973, A\&A 26, 443

Swings J.P., 1974, A\&A 34, 333

Swings J.P., 1995 (personal communications)

Swings J.P., Allen D.A., 1971, ApJ 167, L41

Swings J.P., Struve O., 1943, ApJ 98, 91

Swings J.P., Barbier R., Klutz M., Surdej A., Surdej J., 1980, A\&A 90, 116

Swings J.P., Swings P., 1972, A\&A 17, 142

Thé P.S., de Winter D., Pérez M.R., 1994, A\&AS 104, 315

Voshchinnikov N.V., Molster F.J., Thé P.S., 1995, Ap\&SS 224, 223

Wackerling L.R., 1970, Mem. RAS 73, 153

de Winter D., van den Ancker M.E., Pérez M.R., et al., 1996, A\&AS (in press) (Paper I)

de Winter D., Grinin V.P., Grady C.A., et al., 1995, in "Circumstellar Dust and Planet Formation", Ferlet R. and Vidal-Madjar A. (eds.). Editions Frontières, p. 171

de Winter D., 1996, PhD Thesis, University of Amsterdam

Zickgraf F.-J., Wolf B., Stahl O., Leitherer C. Appenzeller I., 1986, A\&A 163, 119

Zickgraf F.-J., Schulte-Ladbeck R.E., 1989, A\&A 214, 274 